\title{
1 Macrophage-derived insulin/IGF antagonist ImpL2 regulates systemic metabolism for 2 mounting an effective acute immune response in Drosophila
}

3 Krejčová Gabriela ${ }^{1 \#}$, Bajgar Adam ${ }^{1{ }^{1 *}}$, Nedbalová Pavla ${ }^{1}$, Kovářová Julie $^{2}$, Kamps-Hughes Nick $^{3}$,

4 Zemanová Helena ${ }^{1}$, Strych Lukáš ${ }^{1}$, Doležal Tomás ${ }^{1 *}$

$5{ }^{1}$ University of South Bohemia, Czech Republic, ${ }^{2}$ Biology Centre CAS, Czech Republic, ${ }^{3}$ University of Oregon,

6 United States

7 " equal contribution

8 *For correspondence: AB - bajgaa00@prf.jcu.cz TD - tomas.dolezal@prf.jcu.cz

\section{Abstract}

In response to invading pathogens, macrophages metabolically polarize toward Hif1 $\alpha$-induced aerobic glycolysis, requiring increased supply of nutrients. Here, we show that in order to obtain sufficient resources, Drosophila macrophages release the insulin/IGF antagonist ImpL2 in a Hif1 $\alpha$ dependent manner as a reflection of adopted polarization. ImpL2 remotely induces Foxo-mediated lipid mobilization and the release of lipoproteins and carbohydrates from adipose tissue to be utilized by the activated immune system. Although these $1 \mathrm{mpL} 2$ effects are essential in acute streptococcal infection, they become maladaptive during chronic intracellular Listeria infection. The relevance of our model to mammalian immuno-metabolism is documented by the increased expression of the ImpL2 homolog IGFBP7 in human macrophages exposed to Streptococcus. Therefore, we postulate that $1 \mathrm{mpL} /$ /IGFBP7 induce resource mobilization, which is beneficial during the acute phase of infection but may act as cachectic factors in patients with cancer, sepsis, or obesity.

Keywords:

Macrophage, immuno-metabolism, Drosophila, immunity, bacterial infection, Streptococcus, ImpL2, IGFBP7, energy mobilization, Hif1 $\alpha$, aerobic glycolysis, macrophage polarization, insulin resistance, selfish immune system, adipose tissue remodeling, Foxo, infection-induced insulin resistance, Listeria, Insulin/IGF antagonist, wasting, cachexia

\section{Introduction}

Macrophages represent the front line in defence against invading pathogens. Although the effectiveness of the immune response correlates with the number of immune cells (Nicholson and Nicholson 2008), their maintenance requires energy and excessive activation can lead to a myriad of pathologies and metabolic disorders (Shattuck-Heidorn et al. 2016; Zmora et al. 2017). Animals have therefore developed a strategy that allows them to maintain a sufficient number of quiescent immune cells that can be rapidly activated in response to the detection of pathogen- or dangerassociated molecular patterns (Kelly and O'Neill 2015). Numerous populations of sentinel macrophages wait for activation stimuli without being an energy burden. As a consequence, macrophages depend on rapid and sufficient supplementation from external sources, making the acute phase of the immune response challenging for the whole organism (Newsholme et al. 1986). metabolism to generate enough energy and precursors to support their bactericidal function. Therefore, bactericidal (M1) macrophages substantially increase the rate of glycolysis and the pentose phosphate pathway and rewire their mitochondrial metabolism in a Hif1 $\alpha$-dependent 
manner (Van den Bossche, O'Neill, and Menon 2017). Such a metabolic setup resembles the Warburg effect originally described as a unique metabolic program for cancer cells (Warburg 1925). We have recently shown that the metabolic polarization of macrophages is an ancient and evolutionarily conserved process, since even Drosophila macrophages undergo a metabolic switch triggered by Hif1 $\alpha$, which is essential for their bactericidal function (Krejčová et al. 2019).

An adverse aspect of $\mathrm{M} 1$ polarization is that these cells require more energy and become functionally dependent on external sources of glucose, glutamine, and lipids. Therefore, in response to their metabolic activation, macrophages release signals that regulate systemic metabolism and thus ensure their supply of nutrients at the expense of other organs (Straub 2014). Such privileged behavior, in which macrophages usurp nutrients from other processes, is crucial for an effective immune response (Bajgar and Dolezal 2018). One of the factors mediating such behavior is extracellular adenosine, which links the current metabolic state of activated immune cells to the systemic mobilization of carbohydrates, which serve as a source for immune defense (Bajgar et al. 2015; Bajgar and Dolezal 2018). However, the response is very complex and we assume the existence of other signaling factors with an analogous function.

To discover other signaling factors that are released by activated immune cells and regulate systemic metabolism, we sought inspiration in neoplastic tumor research. This idea is based on the notion that both tumors and activated immune cells share commonthe features of their cellular metabolism because both use Hif1 $\alpha$-triggered aerobic glycolysis as the predominant metabolic program (Biswas and Mantovani 2012; Nagao et al. 2019). They also share the impact on systemic metabolism, as both cancer patients and septic patients exhibit a wasting-like phenotype caused by cytokine-induced insulin resistance (Dev, Bruera, and Dalal 2018). Although insulin resistance is mostly studied as a pathological condition, its evolutionary conservation indicates that it must carry an important physiological function (Soeters and Soeters 2012; Odegaard and Chawla 2013). Hence, we hypothesize that activated immune cells may release the same factors as cancer cells, but with a beneficial role for the acute response as opposed to cancer-induced cachexia.

In this study, we focus on the insulin/IGF antagonist ImpL2 (Imaginal morphogenesis protein-Late 2) as a potential candidate for such a role since it is released by neoplastic cancer cells to suppress insulin signaling via binding to Drosophila insulin-like peptides thus causing energy wasting (Alee 2011; Arquier et al. 2006; Honegger et al. 2008; Kwon et al. 2015; Figueroa-Clarevega and Bilder 2015). Besides the production by neoplastic tumors, ImpL2 is known to be released by lipidoverloaded macrophages (Morgantini et al. 2019), as well as other cells employing metabolic programs dependent on the activity of Hif1 $\alpha$ in Drosophila (Alee 2011; Owusu-Ansah, Song, and Perrimon 2013; Kwon et al. 2015; Figueroa-Clarevega and Bilder 2015). Therefore, we decided to test the role of $I \mathrm{mpL} 2$ as a macrophage-derived signaling factor responsible for nutrient mobilization during the acute immune response to bacterial infection.

We show here that activated macrophages produce $I m p L 2$ in the acute phase of infection, resulting in Foxo-mediated changes in adipose tissue metabolism. As an outcome of the $1 \mathrm{mpL} 2$ action, we observed an increased titer of circulating carbohydrates and lipids as well as their accumulation in macrophages. The role of ImpL2 is necessary for effective resistance to streptococcal infection and effective phagocytosis. In contrast to this beneficial role in the fight against extracellular pathogens, the effects of $1 \mathrm{mpL} 2$ are maladaptive in response to intracellularly growing Listeria, and its chronic activation leads to developmental defects and excessive melanization. Conservation of $1 \mathrm{mpL} 2$ function between insects and mammals is indicated by increased expression of the ImpL2 homolog IGFBP7 in streptococcus-treated human macrophages.

\section{Results}

ImpL2 expression increases in immune-activated macrophages in a Hif1 $\alpha$-dependent manner 
93
To test the potential role of $I \mathrm{mpL} 2$ during infection, we first monitored its expression profile during the acute phase of Streptococcus pneumoniae infection. $1 \mathrm{mpL} 2$ expression increased significantly in infected flies compared to PBS-injected controls, starting as early as 3 hours post-infection (hpi) and reaching up to a 3 -fold increase in expression $21 \mathrm{hpi}$ at the whole organism level (Figure 1A). We employed a transcript-specific ImpL2-RA Gal4 driver (Bader et al. 2013) to drive the fluorescent marker UAS-mCherry to identify tissues responsible for infection-induced rise in ImpL2 expression. The pattern of $I \mathrm{mpL2}$-positive cells resembled the characteristic distribution of macrophages in adult flies (Figure 1B). Moreover, the number of these mCherry-expressing cells increased substantially upon infection (Figure $1 \mathrm{~B}$ and $\mathrm{E}$ ). To verify that the cells expressing ImpL2-mCherry are phagocytes, we injected the flies with the phagocytic marker $S$. aureus-pHrodo-Green and it indeed co-localized with these cells (Figure $1 C$ ). In addition, cells expressing $I \mathrm{mpL} 2$ also actively recognized and engulfed S. pneumoniae bacteria ex vivo (Figure 1D). The fact that ImpL2 production induced by infection is specific for macrophages is supported by its increased expression in FACS-sorted CrqGal4>UAS-GFPlabeled macrophages (Figure 1F).

Since there are three alternative transcriptional start sites for $I m p L 2$, we decided to analyze the expression pattern of each isoform $24 \mathrm{hpi}$ to better understand the regulation of their expression. Consistent with the expression pattern of the ImpL2-RA transcript-specific Gal4 driver (Figure 1B), ImpL2-RA transcript expression was the highest of all transcript variants in macrophages and was hardly detectable in fat body or muscles (Figure 1G). While the $1 \mathrm{mpL2}-R B+D$ forms were the most abundant variants in adipose tissue, their expression was five-times weaker than ImpL2-RA in macrophages (check the different $y$-axis scales in Figure 1G). In addition, the expression of the $1 \mathrm{mpL} 2$ $R A$ isoform increased eightfold in macrophages, indicating the most significant increase in expression compared to other transcripts in response to infection (Figure $1 \mathrm{G}$ ). The ImpL2-RA transcript variant expressed in macrophages is therefore a major contributor to the overall increase in $1 \mathrm{mpL2}$ expression observed at the onset of infection.

The previously characterized metabolic switch of activated macrophages is regulated by the transcriptional factor Hif1 $\alpha$ (Krejčová et al. 2019), which is known to be a potent regulator of ImpL2 expression in cells relying on anaerobic metabolism (Allee 2011, Li et al. 2013; Owusu-Ansah, Song, and Perrimon 2013). We, therefore, hypothesized that the same regulation could underlie the infection-induced rise in ImpL2 in activated macrophages. Macrophage-specific knockdown of Hif1 $\alpha$ during infection (see Figure 1-figure supplement $1 \mathrm{~A}$ for efficiency) resulted in the inability of these cells to trigger characteristic infection-induced ImpL2 expression, and this effect was particularly evident in the ImpL2-RA isoform (Figure $1 \mathrm{H}$ ). We identified a cluster of four Hif1 $\alpha$ binding sites upstream of the macrophage-specific transcription start site of $I m p L 2-R A$ (Figure 1I). In addition, this enhancer region shows the strongest hypoxic induction of any sequence surrounding the $1 \mathrm{mpL} 2$ gene (Kamps-Hughes et al. 2015 and Figure 1I), suggesting that Hif1 $\alpha$ may directly drive the transcription of ImpL2-RA in macrophages. In-depth analysis of this 500-bp region revealed the presence of eight immune and stress response elements that cluster together (four hypoxia response elements, two Relish bindings sites, and two heat-shock factor binding sites; Figure 11 and Figure 1-figure supplement 2).

These experiments identified activated macrophages as the predominant producers of ImpL2 in infected adult flies. The increase of ImpL2 production is associated with the metabolic switch induced by Hif1 $\alpha$ in macrophages, presumably by direct binding of Hif1 $\alpha$ and other stress-related transcription factors to the regulatory sequence of the ImpL2-RA isoform. 


\section{Macrophage-derived ImpL2 is required for mobilization of reserves to be utilized by macrophages}

Activated macrophages release signaling factors to mobilize reserves to provide sufficient nutrients for the activated immune system. We show here that macrophages increase $1 \mathrm{mpL} 2$ production during infection, and because $\mathrm{mpL} 2$ was previously associated with reserve mobilization that led to wasting in flies with cancer, we further tested the impact of macrophage-derived $1 \mathrm{mpL} 2$ on systemic metabolism.

For this purpose, we employed Drosophila genetic tools for either macrophage-specific (CrqGal4) or macrophage-specific and time-restricted ( $\mathrm{HmIGal4}$, Gal80 ${ }^{\mathrm{TS}}$ ) manipulation of $\mathrm{ImpL2}$ expression. Using conventional constructs for $\operatorname{ImpL2}$ knockdown (ImpL2 ${ }^{\text {RNAi }}$ ) and overexpression $\left(\operatorname{ImpL2}{ }^{\mathrm{CDS}}\right.$ ), we achieved significant changes in ImpL2 expression (see Figure 1-figure supplement 1B for efficiency). Such experimental manipulations allowed us, on the one hand, to prevent upregulation of $1 \mathrm{mpL} 2$ in macrophages induced by infection and, on the other hand, to simulate an increase of ImpL2 in uninfected individuals.

Both infection and overexpression of $I \mathrm{mpL} 2$ in macrophages significantly reduced triglyceride content in whole flies (Figure 2A), accompanied by dramatic changes in adipose tissue morphology (Figure 2B-E). The average size of lipid droplets decreased with increasing number (Figure 2B, C, and E). The overall area of adipose tissue occupied by lipid droplets was markedly smaller (Figure 2D). It should be emphasized that these infection-induced effects were suppressed by macrophage-specific $1 \mathrm{mpL} 2$ knockdown (Figure 2B-E). Detailed lipidomic analysis of adipose tissue by mass spectrometry revealed that both infection and macrophage-specific overexpression of $I \mathrm{mpL} 2$ cause a proportional shift of lipid content from storage lipid forms (triglycerides) to polar lipid species (phosphatidylethanolamine, phosphatidylinositol) participating in lipid mobilization and transport (Figure 2F). These effects were hampered by ImpL2 knockdown (Figure 2F). Similar effects were also observed for glycogen stores when both infection and macrophage-specific overexpression of ImpL2 significantly reduced glycogen content in whole flies, and knockdown of ImpL2 suppressed this effect (Figure $2 \mathrm{G}$ ). These data thus indicate that macrophage-derived ImpL2 may serve as a mediator of crosstalk between the immune system and adipose tissue.

Besides substantially increased lipolytic and glycogenolytic programs in the adipose tissue of infected individuals, we also observed enhanced autophagy in the adipose tissue of Atg8a-mCherry-bearing flies (Figure 3A), further implying an increase in catabolic metabolism. These metabolic changes induced by infection are consistent with the expression pattern of many metabolic genes in adipose tissue. The MTP, apoLPP, apoLTP, and Bmm genes associated with lipid mobilization, as well as the autophagy-associated Atg1 and Atg6 genes, all increased in adipose tissue during infection (Figure 3B and Figure 3-figure supplement 1) and were under the control of macrophage-derived ImpL2. Indeed, ImpL2 knockdown suppressed this effect, while ImpL2 overexpression mimicked the infection-induced response (Figure 3B and Figure 3-figure supplement 1). A remarkable number of these genes are known to be Foxo targets. The transcriptional activity of Foxo is regulated by insulin signaling and because $1 \mathrm{mpL2}$ is an antagonist of insulin signaling, we analyzed the impact of macrophage-derived ImpL2 on the subcellular localization of Foxo in adipose tissue. We found that Foxo was clearly localized to the nucleus upon infection, while remaining mostly in the cytoplasm of adipocytes of uninfected flies (Figure 4). Macrophage-specific overexpression of ImpL2 recapitulated the infection-induced pattern and, conversely, knockdown reversed the effect of infection on Foxo localization (Figure 4); these effects of $1 \mathrm{mpL2}$ on Foxo localization are in agreement with the expression of Foxo target genes (Figure 3B and Figure 3-figure supplement 1).

Stimulated catabolism of glycogen and triglycerides is manifested by hyperglycemia and hyperlipidemia in the circulation of infected flies when increased titers of glucose, trehalose, and cholesteryl-ester were detected in their hemolymph (Figure 5A-D). Accordingly, infection-activated macrophages increased the accumulation of these energy-rich compounds and cholesterol as a cholesteryl-ester derivative (Figure $5 \mathrm{E}-\mathrm{H}$ ). These results are supported by the increased occurrence of 
lipid droplets in the cytosol of macrophages activated by infection and stained with the neutral lipid dye OilRedO (Figure $5 \mathrm{I}$ and J). The ability of activated macrophages to recognize and uptake lipoproteins was verified by injection of labeled human lipoproteins (LDL-pHrodo; Figure 5K) that can be endocytosed by the activity of the scavenger receptor croquemort (fly homolog of CD36), which is abundantly expressed in Drosophila macrophages. The mobilization of reserves, which leads to an increase in the amount of nutrients in the circulation and subsequently to their increased uptake by macrophages, depends on the production of ImpL2 by macrophages. Indeed, macrophage-specific ImpL2 knockdown suppresses all observed effects stimulated by infection, while ImpL2 overexpression mimics these effects even in uninfected individuals (Figure 5A-I).

Combining all these results, we can conclude that $\operatorname{ImpL2}$, produced by macrophages during infection, triggers a Foxo-mediated transcriptional program in adipose tissue that provides macrophages with the necessary nutrients.

\section{ImpL2 is required for an effective immune response but can be detrimental during chronic} infection

The efficiency of the immune response depends on the supply of sufficient energy and the necessary precursors to the activated immune cells. Therefore, we decided to investigate whether the $1 \mathrm{mpL2}$ mediated metabolic supplementation for macrophages is essential for mounting an adequate immune response. The lack of $1 \mathrm{mpL} 2$ produced by macrophages significantly reduced the survival of S. pneumoniae-infected flies (Figure 6A), which was reflected in the elevated pathogen load (Figure $6 \mathrm{~B}$ ), both indicating a decreased resistance in these individuals. This notion was further supported by the reduced phagocytic rate in these flies (Figure 6E and F). Interestingly, overexpression of ImpL2 24 hours prior to infection improved the resistance of these individuals to streptococcal infection, as evidenced by lower pathogen load and improved survival (Figure $6 \mathrm{C}$ and $\mathrm{D}$ ). These observations suggest that $I \mathrm{mpL} 2$, produced by metabolically activated acute phase macrophages, is essential for systemic metabolic changes that support their efficient bactericidal function.

Although our results demonstrate a beneficial role of $1 \mathrm{mpL} 2$ during acute infection, previous work showing the pathological role of ImpL2 in cancer-induced cachexia (Kwon et al. 2015) suggests that the beneficial role of $I \mathrm{mpL} 2$ may be restricted to a short-time window of acute response. Therefore, we tested the effects of ImpL2 overexpression in macrophages over the lifespan of developing individuals and also challenged flies with chronic infection caused by the intracellular pathogen Listeria monocytogenes. Interestingly, the mere transient overexpression of $1 \mathrm{mpL} 2$ in macrophages led to a frequent occurrence of melanized spots (Figure 7A); even more severe outcomes were achieved by overexpression of $I \mathrm{mpL} 2$ during development, resulting in a smaller adult size (data not shown; similar result to Honegger et al. 2008) accompanied by impaired dorsal closure in roughly half of the individuals (Figure 7A). In addition, overexpression of $1 \mathrm{mpL2}$ reduced resistance to $L$. monocytogenes infection with chronically increased pathogen burden (Figure 7D and E), suggesting that the enhanced effects of $I \mathrm{mpL2}$, in contrast to the acute response, are detrimental in chronic infection. Consistent with this result, suppression of $\mathrm{ImpL2}$ production improved survival and significantly reduced bacterial load during this chronic type of infection (Figure 7B and C).

In conclusion, ImpL2-mediated reallocation of resources to macrophages is essential for an adequate antibacterial immune response and resistance to infection. On the other hand, chronic or excessive production of ImpL2 can cause developmental defects and rather enhance the deleterious effects of chronic infections.

Gene expression of ImpL2 mammalian homolog IGFBP7 is increased in immune-activated THP-1 cells 
We have previously shown that IGFBP7, the mammalian homologue of ImpL2, is produced by lipidloaded liver macrophages in response to feeding a high fat diet, with consequent effects on systemic metabolism through the regulation of insulin signaling in hepatocytes. Notably, ImpL2 released from lipid-loaded macrophages appears to play an analogous role in obese Drosophila (Morgantini et al. 2019). To test the relevance of the role of $1 \mathrm{mpL} 2$ in humans during infection, we measured the expression of IGFBPs in PMA-activated THP-1 human monocytic cell line 24 hours after exposure to $S$. pneumoniae bacteria (Figure 8A). The expression of IGFBP1, IGFBP2, IGFBP5, and IGFBP6 was not detected, indicating that these genes are not expressed in these cells (data not shown). Interestingly, IGFBP7 gene expression was significantly increased in inoculated culture compared to PBS-treated controls (Figure $8 \mathrm{~B}$ ). In addition, gene expression of anti-inflammatory IGFBP3 was reduced four-fold while IGFBP4 showed no significant change (Figure $8 \mathrm{~B}$ ). These data therefore indicate that the increase in ImpL2 / IGFBP7 production by macrophages in response to infection is evolutionarily conserved in mammals. These results not only validate the importance of the acute bacterial infection model in Drosophila but also suggest that investigating the role of IGFBP7 in systemic metabolic regulation and inter-organ communication during infection would be of interest in humans.

\section{Discussion}

In this work, we show that infection-driven Hif1 $\alpha$ transcriptional activity connects intracellular metabolic switch with $1 \mathrm{mpL} 2$ production in activated macrophages. $1 \mathrm{mpL} 2$ subsequently induces the mobilization of adipose tissue resources to be provided for activated immune cells. This metabolic rearrangement of adipose tissue is mediated by the transcription factor Foxo, which results in the acceleration of lipolysis and assembly of lipoproteins. The increased titer of circulating lipids facilitates the utilization of lipids by macrophages and thus supports their bactericidal function (Figure 9). Although such ImpL2-induced metabolic adaptation is essential for an acute immune response, it can become maladaptive in cases of its chronic activation or infection by intracellular pathogens that metabolically exploit the host cell. This can be considered a remarkable example of how a beneficial metabolic program can become maladaptive in chronic diseases. That this mechanism might not be limited to insects is indicated by an experiment showing that human macrophages activated by the same bacteria increase the expression of IGFBP7, the human homolog of Drosophila ImpL2.

We have previously shown that Drosophila macrophages undergo evolutionarily conserved polarization to the M1 phenotype, thus adjusting their metabolism toward Hif $1 \alpha$-induced aerobic glycolysis in response to bacterial challenge. This cellular metabolic shift is accompanied by systemic metabolic changes necessary to supplement the sudden needs of macrophages (Krejčová et al. 2019). Coordination of cellular metabolism in macrophages with systemic metabolism is crucial for resistance to infection and anticipates the existence of circulating factors mediating this inter-organ crosstalk (Dolezal et al. 2019). Inspired by research on neoplastic cancer cells (Kwon et al. 2015; Figueroa-Clarevega and Bilder 2015; Bunker et al. 2015), we focus in this study on the cachectic factor ImpL2 as a candidate previously linked to Hif1 $\alpha$ transcriptional activity while having the potential to regulate systemic metabolism (Alee 2011; Li et al. 2013). To test whether the interconnection between Hif1 $\alpha$ activity and $1 \mathrm{mpL} 2$ expression during hypoxia also applies to infection-activated macrophages, we performed a genomic in silico meta-analysis of hypoxic enhancer activity and Hif1 $\alpha$ binding sites (Kamps-Hughes et al. 2015). This approach revealed the induction of a hypoxic enhancer in close vicinity to the transcription start site of the $\operatorname{ImpL2-RA}$ transcript variant, which is further supported by the presence of four hypoxia response elements at this locus. ImpL2-RA is the most strongly expressed transcriptional variant in macrophages and its expression increases even more after infection. This infection-induced increase depends on Hif1 $\alpha$, 
283 indicating a connection of ImpL2 expression with an internal metabolic switch in infection284 activated macrophages.

285 We have previously identified extracellular adenosine as an immune cell-derived systemic factor with an analogous function to ImpL2 in mobilizing carbohydrate stores to be available to the immune system (Bajgar et al. 2015; Bajgar and Dolezal 2018). In addition to regulating carbohydrate metabolism, a prominent effect of $1 \mathrm{mpL} 2$ is the regulation of systemic lipid metabolism. As documented in this work by various approaches, macrophage-derived ImpL2 mediates infectioninduced catabolic processes in adipose tissue resulting in the assembly and release of lipoproteins for the redistribution of resources to metabolically demanding tissues. Thus, infection-induced ImpL2 production results in an increased titer of circulating lipids, which are then used by macrophages to effectively fight invading pathogens. The observed metabolic changes in infectionchallenged flies resemble lipemia as a characteristic symptom of sepsis in severely ill patients (Harris, Gosnell, and Kumwenda 2000). In sepsis-induced lipemia, hepatocytes release low-density lipoproteins (LDL) and very low-density lipoproteins (VLDL), providing lipids to be preferentially utilized by bactericidal macrophages in the periphery (Khovidhunkit et al. 2004; Aspichueta et al. 2012). Scavenger receptor-mediated endocytosis of serum lipoproteins, together with attenuated reverse cholesterol transport, both contribute substantially to cholesterol accumulation in bactericidal immune cells (Podrez et al. 2000). There are many immune-related functions in macrophages dependent on sufficient delivery of lipids, from phagocytosis and maturation of phagolysosome to catecholamine production or immune memory formation via the mevalonate pathway (summarized in Remmerie and Scott 2018). Although providing substantial amounts of lipids to macrophages is essential for their proper function during the acute phase of infection, chronic exposure of macrophages to excessive lipids may lead to the adoption of a foam-cell phenotype promoting metabolic syndrome and atherosclerosis (Chistiakov et al. 2017; Febbraio, Guy, and Silverstein 2004).

Although the mechanism of infection-induced lipid catabolism and lipoprotein assembly has not been fully elucidated, we revealed that nuclear translocation of Foxo, followed by increased expression of several Foxo-target genes involved in this process, was regulated by macrophage-derived ImpL2. Since Foxo-driven mobilization of lipid stores requires suppression of insulin signaling in adipose tissue (Molaei, Vandehoef, and Karpac 2019; Luong et al. 2006), the effect of ImpL2 can be explained by its well-documented ability to bind Drosophila insulin-like peptides (DILPS) and thus reduce insulin sensitivity in adipose tissue (Alee 2011; Alic et al. 2011; Arquier et al. 2006; Figueroa-Clarevega and Bilder 2015; Honegger et al. 2008; Kwon et al. 2015; Okamoto et al. 2013). Metabolic adjustments and the switch from anabolism to catabolism in adipose tissue during infection is regulated by multiple mechanisms (reviewed in Dolezal et al. 2019). In particular, Foxo has previously been associated with wasting in flies with chronic infection (Dionne et al. 2006), indicating rather its pathological effects during the prolonged immune response. Based on the aforementioned, the simplest explanation for the effect of $I \mathrm{mpL2}$ on the mobilization of adipose tissue sources is the suppression of insulin signaling to allow a metabolic program triggered by Foxo. The remote ImpL2 effect on reserve mobilization described in this work therefore represents a beneficial role of ImpL2 during the acute immune response. Suppression of insulin signaling is often associated with chronic inflammation and may become harmful to the organism by disrupting the metabolic balance. However, the theoretical concept of selfish immunity (Straub 2014) sees insulin resistance as an evolutionary adaptive mechanism for rerouting nutrients toward immunity during the acute immune response. Our results showing that $I \mathrm{mpL} 2$ is required for an effective immune response through changes in lipid metabolism can be considered as experimental evidence of insulin resistance as an adaptive mechanism for mobilization of sources for acute phase of immune response.

Neoplastic tumors share a characteristic cellular metabolism (Warburg 1925) with bactericidal macrophages (Andrejeva and Rathmell 2017). Here, we show that they may also share the production of ImpL2, originally perceived as a cancer-derived cachectic factor (Kwon et al. 2015; 
333 Figueroa-Clarevega and Bilder 2015), to regulate systemic metabolism. Both tumor cells and activated immune cells produce $\mathrm{ImpL2}$ and thus subsequently silence systemic insulin signaling as a result of the adoption of Hif1 $\alpha$-triggered aerobic glycolysis. The perception of $I \mathrm{mpL} 2$ expression as a consequence of the cellular metabolic state is further supported by other conditions of mitochondria-independent generation of ATP, such as experimentally induced mitochondrial dysfunction, hypoxia, and neoplastic tumor growth (Alee 2011; Figueroa-Clarevega and Bilder 2015; Kwon et al. 2015; Li et al. 2013). Although this mechanism is beneficial to the organism in acute infection, it becomes harmful in the case of tumor growth or chronic infection, as demonstrated here by the negative effect of ImpL2 on survival during chronic infection caused by the intracellular pathogen $L$. monocytogenes. This can be explained by the known behavior of various intracellular bacteria that use the supply of host cells and hijack their metabolic cascades to literarily nourish themselves (Péan et al. 2017; Teng, Ang, and Guan 2017).

Our results revealed that human macrophages, activated by $S$. pneumoniae, increase the expression of IGFBP7, the human homolog of Drosophila ImpL2, suggesting that a similar mechanism may function in humans. Notably, not only infection but also experimentally induced metabolic syndrome in both Drosophila and mammals led to increased expression of $1 \mathrm{mpL2} / \mathrm{IGFBP7}$ in macrophages (Morgantini et al. 2019). While ImpL2-producing macrophages affect metabolism in the Drosophila fat body (integrating functions of both mammalian adipose tissue and liver), IGFBP7-producing Kupffer cells act directly on hepatocytes in order to induce systemic metabolic changes (Morgantini et al. 2019; Akiel et al. 2017). The ability of both ImpL2 and IGFBP7 to bind extracellular insulin, thereby reducing systemic insulin signaling, further documents their functional homology, suggesting that the mechanism of their action may also be conserved (Honegger et al. 2008; Arquier et al. 2006; Oh et al. 1996). Accordingly, LPS-administered Kupffer cells have been shown to produce cytokines to affect Foxo-mediated release of LDL and VLDL from hepatocytes (Kamagate et al. 2008; Sparks and Dong 2009; Aspichueta et al. 2012). Although this mechanism of resource mobilization may be important for the acute phase response, when prolonged, it contributes to the progression of metabolic syndrome and atherosclerosis (Tomkin 2012). It is therefore not surprising that the levels of circulating IGFBP7 are considered a marker of systemic metabolic imbalances accompanying several human diseases, such as obesity, acute kidney injury, liver fibrosis, and chronic obstructive pulmonary disease (Liu et al. 2015; Gunnerson et al. 2016; Martínez-Castillo et al. 2020; Ruan et al. 2017).

364 Taking our data together with previous work, we can conclude that the insulin/IGF antagonist ImpL2, released from M1 macrophages as a reflection of their metabolic polarization, mediates the mobilization of nutrients from adipose tissue by inducing insulin resistance to provide sufficient resources for the immune system. Despite the fact that both $\mathrm{ImpL2}$ and insulin resistance are mostly studied in the context of pathological conditions such as cancer-induced cachexia, obesity and chronic inflammatory conditions, our data revealed that they may play a beneficial role in the acute phase of bacterial infection. In addition, the relevance of our model is supported by the production of IGFBP7, the ImpL2 homolog, in response to streptococcal infection in human macrophages. This leads us to the hypothesis that analogous mechanisms may also apply to macrophage-induced nutrient mobilization from hepatocytes during the acute phase of the immune response, which may become detrimental under chronic conditions.

Funding

377 Tomáš Doležal - Czech Science Foundation (Project 20-09103S)

378 Adam Bajgar - Czech Science Foundation (Project 20-14030S)

379 Gabriela Krejčová - USB Grant Agency (Project 050/2019/P) 


\section{Acknowledgment}

The authors acknowledge funding from the Grant Agency of the Czech Republic to TD (Project 20091035; www.gacr.cz) and to AB (Project 20-14030S; www.gacr.cz). GK was supported by USB Grant Agency (Project 050/2019/P). We thank to Lucie Hrádková for laboratory services, enthusiasm and support, Alena Krejčí-Bruce and Lenka Chodáková for critical comments and inspiring discussions, Pavel Branný and Linda Doubravová for help with the preparation of the S.p.-GFP strain and Hana Sehadová for help with scanning electron microscopy. We thank to Hugo Stocker for the $1 \mathrm{mpL2}{ }^{\text {RNAi }}$, ImpL2 ${ }^{\mathrm{CDS}}$, and ImpL2-RA-Gal4 fly lines, Gabor Juhasz for the Atg8amCherry fly, and Marc Dionne for Crq>GFP fly line. Other fly stocks were obtained from the Bloomington Center (Bloomington, IN) and the VDRC (Vienna, Austria). The S. pneumoniae and L. monocytogenes bacterial strains were obtained from Dr. David Schneider. We also thank Martin Moss and Petr Šimek for the lipidomics service, the Department of Medical Biology (USB) for allowing us to use the S3eBioRad sorter, Biology Centre CAS for allowing us to use a confocal microscope, and a laboratory equipped to maintain human tissue cultures. We are also grateful to developers of Fiji: an open-source platform for biological-image analysis (doi:10.1038/nmeth.2019)

\section{MATERIALS AND METHODS}

\section{Drosophila melanogaster strains}

The flies were raised on a diet containing cornmeal $(80 \mathrm{~g} / \mathrm{l})$, sucrose $(50 \mathrm{~g} / \mathrm{l})$, yeast $(40 \mathrm{~g} / \mathrm{l})$, agar $(10$ $\mathrm{g} / \mathrm{l})$, and $10 \%$-methylparaben $(16.7 \mathrm{~mL} / \mathrm{l})$ and maintained in a humidity-controlled environment with a natural $12 \mathrm{~h} / 12 \mathrm{~h}$ light/dark cycle at $25^{\circ} \mathrm{C}$. Flies carrying Gal80 protein were raised at $18^{\circ} \mathrm{C}$ and transferred to $29^{\circ} \mathrm{C} 24 \mathrm{~h}$ prior to infection in order to degrade temperature-sensitive Gal80. Prior to the experiments, flies were kept in plastic vials on a sucrose-free cornmeal diet (cornmeal $53.5 \mathrm{~g} / \mathrm{l}$, yeast $28.2 \mathrm{~g} / \mathrm{l}$, agar $6.2 \mathrm{~g} / \mathrm{l}$ and $10 \%$-methylparaben $16.7 \mathrm{~mL} / \mathrm{l}$ ) for 7 days. Flies infected with $\mathrm{S}$. pneumoniae were kept on a sucrose-free cornmeal diet in incubators at $29^{\circ} \mathrm{C}$ due to the temperature sensitivity of $S$. pneumoniae. They were transferred to fresh vials every other day without the use of $\mathrm{CO}_{2}$ to ensure good food condition. Flies infected with $L$. monocytogenes were kept on a sucrose-free cornmeal diet at $25^{\circ} \mathrm{C}$. The Drosophila Stock Center in Bloomington provided $I m p L 2^{R N A i}$ ( $y 1 s C^{*} v 1$; P\{TRiP.HMC03863\}attP40; FBst0055855) TRiP ${ }^{\text {control }}$ (y[1] v[1] ; P\{y[+t7.7]=CaryP\}attP40; FBst0036304) and 20xUAS-6xmCherry (P\{20XUAS-6XmCherry-HA\}attP2; FBtp0094992) flies. ImpL2-Gal4 (ImpL2-RAGal4; FBal0290965) and UAS-ImpL2 ${ }^{\text {cds }}$ (UAS-s.ImpL2; FBal0249386) were kind gifts from Hugo Stocker. CrqGal4>2xeGFP were obtained from Marc Dionne. The Atg8a-mCherry strain was kindly provided by Gabor Juhasz. The $w^{1118}$ strain has a genetic background based on CantonS.

\section{Genotypes of experimental models}

\section{Figure 1}

ImpL2GaI4>UASmCherry corresponds to +/+; 20xUAS-6xmCherry/+; ImpL2-Gal4/+

HmIGal4>GFP refers to HmIS-Gal4 UAS-2xeGFP/HmIS-Gal4 UAS-2xeGFP; +/+

HmlGal4>GFP; Hif1 $\alpha^{\text {RNAi }}$ corresponds to HmIS-Gal4 UAS-2xeGFP/UAS-Hif $1 \alpha^{R N A i} ;+/+$ 


\section{$421 \quad$ Figure 2}

422

ImpL2 ${ }^{\text {RNAi }}$ refers to $+/+; H m I \Delta-G a l 4 /$ UAS-ImpL2 $2^{\text {RNAi }} ;$ P $\{$ tubPGal80ts $\} /+$

423

ImpL2 ${ }^{\text {cds }}$ corresponds to $+/+; H m / \Delta-G a / 4 /+; P\{$ tubPGal80ts $\} / U A S-I m p L 2^{\text {cds }}$

424

TRiP $^{\text {control }}$ refers to $+/+; H m / \Delta-G a / 4 /+; P\{$ tubPGal80ts $\} / T R i P^{\text {control }}$

425

$\mathbf{W}^{1118}$ corresponds to $w^{1118} /+; H m / \Delta-G a l 4 /+; P\{$ tubPGal80ts $\} /+$

426

427

Figure 3

428

ImpL2 ${ }^{\mathrm{RNAi}}$ refers to $+/+; \mathrm{HmI} \Delta$-Gal4/UAS-ImpL $2^{\text {RNAi}} ;$ P $\{$ tubPGal80ts $\} /+$

ImpL2 ${ }^{\text {cds }}$ corresponds to $+/+; H m / \Delta-G a / 4 /+; P\{$ tubPGal80ts $\} / U A S-I m p L 2^{\text {cds }}$

430

TRiP $^{\text {control }}$ refers to $+/+; H m / \Delta-G a l 4 /+; P\{$ tubPGal80ts $\} /$ TRiP $P^{\text {control }}$

431

$\mathbf{W}^{\mathbf{1 1 1 8}}$ corresponds to $w^{1118} /+; H m / \Delta-G a l 4 /+;$ P $\{$ tubPGal80ts $\} /+$

Atg8a-mCherry refers to gen $+/+;$ Atg $8 a$-mCherry/Atg8a-mCherry

Figure 4

ImpL2 ${ }^{\text {RNAi }}$ refers to $+/+; H m I \Delta-G a l 4 /$ UAS-ImpL2 $2^{\text {RNAi }} ;$ P $\{$ tubPGal80ts $\} /+$

ImpL2 ${ }^{\text {cds }}$ corresponds to $+/+; H m / \Delta-G a / 4 /+; P\{t u b P G a I 80 t s\} / U A S-I m p L 2^{\text {cds }}$

TRiP $^{\text {control }}$ refers to $+/+; H m / \Delta-G a / 4 /+; P\{$ tubPGal80ts $\} / T R i P^{\text {control }}$

438

$\mathbf{W}^{1118}$ corresponds to $w^{1118} /+; H m I \Delta-G a l 4 /+;$ P $\{$ tubPGal80ts $\} /+$

Figure 5

ImpL2 ${ }^{\text {RNAi }}$ refers to $+/+; H m I \Delta-G a l 4 /$ UAS-ImpL $2^{\text {RNAi }} ;$ P $\{$ tubPGal80ts $\} /+$

ImpL2 ${ }^{\text {cds }}$ corresponds to $+/+; H m / \Delta-G a / 4 /+; P\{t u b P G a I 80 t s\} / U A S-I m p L 2^{\text {cds }}$

TRiP $^{\text {control }}$ refers to $+/+; H m / \Delta-G a l 4 /+; P\{$ tubPGal80ts $\} /$ TRiP $P^{\text {control }}$

$\mathbf{W}^{\mathbf{1 1 1 8}}$ corresponds to $w^{1118} /+; H m / \Delta-G a l 4 /+;$ P $\{$ tubPGal80ts $\} /+$

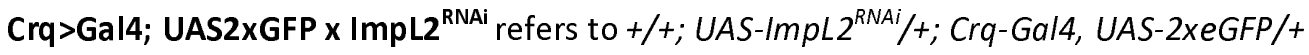

Crq>Gal4; UAS2xGFP $\times$ ImpL2 ${ }^{\text {cds }}$ corresponds to $+/+;+/+$; Crq-Gal4, UAS-2xeGFP/UAS-ImpL2 ${ }^{\text {cds }}$

Crq>Gal4; UAS2xGFP x TRiP ${ }^{\text {control }}$ refers to $+/+;+/+$; Crq-Gal4, UAS-2xeGFP/TRiP control $^{\text {. }}$

Crq>Gal4; UAS2xGFP $\times$ W $^{1118}$ corresponds to $w^{1118} /+$; Crq-Gal4, UAS-2xeGFP/+

CrqGal4>GFP refers to +/+; Crq-Gal4, UAS-2xeGFP/Crq-Gal4, UAS-2xeGFP 
452 ImpL2 ${ }^{\text {RNAi }}$ refers to +/+; HmIS-Gal4/UAS-ImpL2 ${ }^{\text {RNAi }} ;$ P\{tubPGal80ts $\} /+$

453 ImpL2 ${ }^{\text {cds }}$ corresponds to +/+; HmIL-Gal4/+; P\{tubPGal80ts $\} / U A S-I m p L 2^{\text {cds }}$

454 TRiP $^{\text {control }}$ refers to $+/+; H m / \Delta-G a / 4 /+;$ P $\{$ tubPGal80ts $\} / T R i P^{\text {control }}$

$455 \mathbf{W}^{1118}$ corresponds to $w^{1118} /+; H m / \Delta-G a l 4 /+;$ P $\{$ tubPGal80ts $\} /+$

456 Crq>Gal4; UAS2xGFP $\times$ ImpL2 ${ }^{\text {RNAi }}$ refers to +/+; UAS-ImpL2 $2^{\text {RNAi } /+; C r q-G a I 4, ~ U A S-2 x e G F P /+~}$

457 Crq>Gal4; UAS2xGFP $\times$ ImpL2 ${ }^{\text {cds }}$ corresponds to +/+; +/+; Crq-Gal4, UAS-2xeGFP/UAS-ImpL2 $2^{\text {cds }}$

458 Crq>Gal4; UAS2xGFP x TRiP ${ }^{\text {control }}$ refers to $+/+$; +/+; Crq-Gal4, UAS-2xeGFP/TRiP ${ }^{\text {control }}$

459 Crq>Gal4; UAS2xGFP $\times$ W $^{1118}$ corresponds to $w^{1118} /+$; Crq-Gal4, UAS-2xeGFP/+

460 CrqGal4>GFP refers to +/+;Crq-Gal4, UAS-2xeGFP/Crq-Gal4, UAS-2xeGFP

461

Figure 7

ImpL2 ${ }^{\text {RNAi }}$ refers to +/+; HmI 4 -Gal4/ UAS-ImpL2 ${ }^{\text {RNAi }} ;$ P\{tubPGal8Ots $\} /+$

ImpL2 ${ }^{\text {cds }}$ corresponds to +/+; HmIS-Gal4/+; P\{tubPGal80ts $\} / U A S-I m p L 2^{\text {cds }}$

TRiP $^{\text {control }}$ refers to $+/+; H m / \Delta-G a / 4 /+; P\{$ tubPGal80ts $\} / T R i P^{\text {control }}$

$\mathbf{W}^{1118}$ corresponds to $\mathrm{w}^{1118} / \mathrm{H} ; \mathrm{Hm} / \Delta-G a / 4 /+;$ P $\{$ tubPGal8Ots $\} /+$

Crq $>$ Gal4 $\times$ ImpL2 ${ }^{\text {cds }}$ corresponds to $+/+;+/+$; Crq-Gal4, UAS-2xeGFP/UAS-ImpL2 $2^{\text {cds }}$

\section{Hypoxic enhancer activity analysis}

Genome-wide experimental data characterizing hypoxia-induced transcriptional enhancer activity transcriptional induction is defined as the ratio of expressed randomer tag counts in hypoxic versus normoxic conditions and was binned by 500-bp regions across the $I m p L 2$ locus. Each 500-bp bin was then analyzed for transcription factor binding sites corresponding to hypoxic (Hif1 $\alpha$ ), immune (Rel) and stress (HSF) response transcription factors. Transcription factor position frequency matrices were downloaded from the JASPAR database (https://academic.oup.com/nar/article/32/suppl 1/D91/2505159) and queried against the 500-bp bin sequences using BOBRO software (https://www.ncbi.nlm.nih.gov/pmc/articles/PMC3074163/). $B O B R O$ generates a $p$-value of enrichment for the position frequency matrix within the search sequence and also identifies the location of the predicted binding sites.

\section{Gene expression analysis}

Gene expression analyzes were performed on several types of samples: six whole flies, six thoraxes, six fat bodies, or 20,000 isolated macrophages. Macrophages were isolated by a cell sorter as described in (Krejčová et al. 2019) while dissections were made in PBS, transferred to TRIzol Reagent (Invitrogen) and homogenized using a DEPC-treated pestle. Subsequently, RNA was extracted by TRIzol Reagent (Invitrogen) according to the manufacturer's protocol. Superscript III Reverse Transcriptase (Invitrogen) primed by oligo(dT)20 primer was used for reverse transcription. Relative 
489 Rad) using the TP 2x SYBR Master Mix (Top-Bio) in three technical replicates with the following 490 protocol: initial denaturation $-3 \mathrm{~min}$ at $95^{\circ} \mathrm{C}$, amplification $-15 \mathrm{~s}$ at $94^{\circ} \mathrm{C}, 20 \mathrm{~s}$ at $56^{\circ} \mathrm{C}, 25 \mathrm{~s}$ at $72^{\circ} \mathrm{C}$ 491 for 40 cycles. Melting curve analysis was performed at $65-85^{\circ} \mathrm{C} /$ step $0.5^{\circ} \mathrm{C}$. The primer sequences are 492 listed in the Key Resources Table. The qPCR data were analyzed using double delta $\mathrm{Ct}$ analysis, and the expressions or specific genes were normalized to the expression of Ribosomal protein 49 (Rp49) in the corresponding sample. The relative values (fold change) to control are shown in the graphs. Samples for gene expression analysis were collected from three independent experiments. Data were compared with ANOVA followed by Tukey's multiple comparisons test in Graphpad Prism software. Sidak's multiple comparison correction was performed.

\section{Metabolite measurement}

To measure metabolite concentration, isolated macrophages, whole flies or hemolymph were used. Macrophages were isolated from 25 adult males by centrifugation (14,000 RPM, $5 \mathrm{~min}$ ) through a silicagel filter into $50 \mu \mathrm{L}$ of PBS. For measurement of metabolites from whole flies, five flies were homogenized in $200 \mu \mathrm{L}$ of PBS and centrifuged ( $3 \mathrm{~min}, 4^{\circ} \mathrm{C}, 8,000 \mathrm{RPM}$ ) to discard insoluble debris. 50,000 macrophages were isolated by cell sorter as described above. Half of all samples were used for the quantification of proteins. Samples for glucose, glycogen, trehalose, and triglyceride measurement were denatured at $75^{\circ} \mathrm{C}$ for $10 \mathrm{~min}$, whereas samples for protein quantification were frozen immediately in $-80^{\circ} \mathrm{C}$. Glucose was measured using a Glucose (GO) Assay (GAGO-20) Kit (Sigma) according to the manufacturer's protocol. Colorimetric reaction was measured at $540 \mathrm{~nm}$. For glycogen quantification, sample was mixed with amyloglucosidase (Sigma) and incubated at $37^{\circ} \mathrm{C}$ for 30 min. A Bicinchoninic Acid Assay (BCA) Kit (Sigma) was used for protein quantification according to the supplier's protocol and the absorbance was measured at $595 \mathrm{~nm}$. Cholesterol and cholesteryl esters were measured on isolated lipid fraction by using Cholesterol/Cholesteryl Ester Quantitation Kit (Sigma) according to the supplier's protocol. Triglycerides were measured using Triglyceride quantification Colorimetric/Fluorometric Kit (Sigma). For trehalose quantification, sample was mixed with trehalase (Sigma) and incubated at $37^{\circ} \mathrm{C}$ for $30 \mathrm{~min}$. Samples for metabolite concentration were collected from three independent experiments. Measured data were compared in Graphpad Prism using ANOVA followed by Tukey's multiple comparisons test. Sidak's multiple comparison correction 517 was performed.

Flies were dissected in Grace's Insect Medium (Sigma) and subsequently stained with DAPI and Cell Brite Fix Membrane Stain 488 (Biotium) diluted with Grace's Insect Medium according to the manufacturer's protocol at $37^{\circ} \mathrm{C}$. Tissues were washed in PBS and then fixed with $4 \%$ PFA (Polysciences). After 20 min, the tissues were washed in PBS and pre-washed with $60 \%$ isopropanol. Dissected abdomens were then stained with OilRedO dissolved in $60 \%$ isopropanol for $10 \mathrm{~min}$. The tissues were then washed with 60\% isopropanol and mounted in an Aqua Polymount (Polysciences). Tissues were imaged using an Olympus FluoView 3000 confocal microscope (Olympus). Content of lipids in adipose tissue and size of lipid droplets were analyzed using Fiji software. Flies for the analysis of LDs in the fat body were collected from three independent experiments and representative images are shown.

\section{Lipidomic analysis}

530 Adipose tissues from six flies from each group were dissected in ice-cold PBS for lipidomics analysis. 531 The removed tissues were stored in PBS buffer in Eppendorf tubes at $-80^{\circ} \mathrm{C}$. Immediately after 532 thawing, they were extracted by $500 \mu \mathrm{l}$ of cold chloroform: methanol solution ( $\mathrm{v} / \mathrm{v} ; 1: 1)$. The samples 533 were then homogenized by a Tissue Lyser II (Qiagen, Prague, Czech Republic) at $50 \mathrm{~Hz},-18^{\circ} \mathrm{C}$ for 5 
$534 \mathrm{~min}$ and kept further in an ultrasonic bath $\left(0^{\circ} \mathrm{C}, 5 \mathrm{~min}\right)$. Further, the mixture was centrifuged at $53510,000 \mathrm{RPM}$ at $4^{\circ} \mathrm{C}$ for $10 \mathrm{~min}$ followed by the removal of the supernatant. The extraction step was 536 repeated at the same conditions. The lower layer of pooled supernatant was evaporated to dryness 537 under a gentle stream of Argon. The dry total lipid extract was re-dissolved in $500 \mu \mathrm{l}$ of chloroform: 538 methanol solution ( $\mathrm{v} / \mathrm{v} ; 1: 1$ ) and directly measured using previously described methods (Bayley et al. 539 2020). Briefly, high performance liquid chromatography (Accela 600 pump, Accela AS autosampler) 540 combined with mass spectrometry LTQ-XL (all Thermo Fisher Scientific, San Jose, CA, USA) were used. 541 The chromatographic conditions were as follows: Injection volume $5 \mu \mathrm{l}$; column Gemini $3 \mu \mathrm{M}$ C18 542 HPLC column $\left(150 \times 2 \mathrm{~mm}\right.$ ID, Phenomenex, Torrance, CA, USA) at $35^{\circ} \mathrm{C}$; the mobile phase (A) $5 \mathrm{mM}$ 543 ammonium acetate in methanol with ammonia (0.025\%), (B) water and (C) isopropanol: $\mathrm{MeOH}(8: 2)$; 544 gradient change of A:B:C as follows: $0 \mathrm{~min}: 92: 8: 0,7 \mathrm{~min}: 97: 3: 0,12 \mathrm{~min}:$ 100:0:0, $19 \mathrm{~min}: 93: 0: 7,20-$ $54529 \mathrm{~min}$ : 90:0:10, 40-45 $\mathrm{min}: 40: 0: 60,48 \mathrm{~min}:$ 100:0:0, and 50-65 $\mathrm{min}$ : 92:8:0 with flow rate 200 $546 \mu \mathrm{l} / \mathrm{min}$. The mass spectrometry condition: positive $(3 \mathrm{kV})$ and negative $(-2,5 \mathrm{kV})$ ion detection mode; 547 capillary temperature $200^{\circ} \mathrm{C}$. Eluted ions were detected with full scan mode from 200 to $1000 \mathrm{Da}$ 548 with the collisionally induced MS2 fragmentation (NCE 35). Data were acquired and processed by 549 means of XCalibur 4.0 software (Thermo Fisher). The corrected areas under individual analytical 550 peaks were expressed in percentages assuming that the total area of all detected is $100 \%$.

\section{Autophagy visualization}

552 To visualize autophagy in adipose tissue, Atg8a-mCHerry-bearing flies were dissected in PBS, fixed 553 with 4\% PFA, and then washed with PBS and stained with DAPI. Fat bodies were imaged using an 554 Olympus FluoView 3000 confocal microscope (Olympus).

\section{Foxo subcellular localization}

556 Adipose tissues for immunolocalization of Foxo were dissected in ice-cold PBS. Dissection was 557 followed by 20-min fixation in 4\% PFA in PBS. After three washing steps in PBS-Tween (0.1\%), 558 unspecific binding was blocked with $10 \%$ NGS in PBS for $1 \mathrm{~h}$ at RT. The tissues were then incubated 559 with primary antibody diluted in ratio 1:1,000 (Rabbit Drosophila anti-Foxo, CosmoBio) at $4^{\circ} \mathrm{C}$ 560 overnight. After washing the unbound primary antibody (three times for $10 \mathrm{~min}$ in PBST), the 561 secondary antibody was applied in dilution 1:250 for $2 \mathrm{~h}$ at RT (Cy2 Goat anti-Rabbit, Jackson562 Immunoresearch). The nuclei were stained with DAPI. Tissues were mounted with Aqua-Polymount 563 (Polysciences), DAPI was used for nuclear staining. Foxo localization was detected by Plot-Profile 564 analysis in FIJI.

\section{Isolation of macrophages}

566 GFP-labeled macrophages were isolated from Crq-Ga/4, UAS-eGFP male flies using fluorescence567 activated cell sorting (FACS). Approximately 200 flies were anaesthetized with $\mathrm{CO}_{2}$, washed in PBS 568 and homogenized in $600 \mathrm{~mL}$ of PBS using a pestle. The homogenate was sieved through a nylon cell 569 strainer $(40 \mu \mathrm{m})$. This strainer was then additionally washed with $200 \mu \mathrm{L}$ of PBS, which was added to 570 the homogenate subsequently. The samples were centrifuged ( $\left.3 \mathrm{~min}, 4^{\circ} \mathrm{C}, 3,500 \mathrm{RPM}\right)$ and the 571 supernatant was washed with ice-cold PBS after each centrifugation (three times). Prior to sorting, 572 samples were transferred to FACS polystyrene tubes using a disposable bacterial filter $(50 \mu \mathrm{m}$, 573 Sysmex) and macrophages were sorted into $100 \mu \mathrm{L}$ of PBS using a S3 ${ }^{\mathrm{TM}}$ Cell Sorter (BioRad). Isolated 574 cells were verified by fluorescence microscopy and differential interference contrast.

\section{Phagocytic activity}


576 To visualize phagocytic activity, flies were injected with $50 \mathrm{nl}$ of pHrodo ${ }^{\mathrm{TM}}$ Green S. aureus (Thermo 577 Fischer Scientific) using an Eppendorf Femtojet microinjector. After $1 \mathrm{~h}$, the flies were fixed in 4\% PFA 578 for 45 min, washed in PBS and subsequently mounted on imaging dishes using an Aqua Polymount 579 (Polysciences). Thoraxes were imaged using an Olympus FluoView 3000 confocal microscope. To 580 analyze phagocytic rate, flies were infected with 20,000 of $S$. pneumoniae and after $24 \mathrm{~h}$, they were 581 injected with $50 \mathrm{nl}$ of $\mathrm{pHrodo}^{\mathrm{TM}}$ Red S. aureus (Thermo Fischer Scientific). After $1 \mathrm{~h}$, abdomens of flies 582 were opened in PBS and the macrophages were allowed to attach to a microscopic imaging dish for $58340 \mathrm{~min}$. Subsequently, the PBS was removed and the cells were fixed for 20 min with $4 \%$ PFA. Aqua Polymount (Polysciences) was used to mount the sample. Macrophages were imaged using an Olympus FluoView 3000 confocal microscope and red dots depicting phagocytic events were 586 manually counted per cell.

\section{Lipoprotein uptake}

To document the uptake of lipoproteins by Drosophila macrophages, flies were injected with $50 \mathrm{nl}$ of pHrodo $^{T M}$ Red LDL (Thermo Fischer Scientific) using an Eppendorf Femtojet microinjector. After $1 \mathrm{~h}$, abdomens of flies were opened in PBS and the macrophages were allowed to attach to a microscopic imaging dish for $40 \mathrm{~min}$. Subsequently, the PBS was removed and cells were fixed for $20 \mathrm{~min}$ with $4 \%$ PFA. Aqua Polymount (Polysciences) was used to mount the sample. Macrophages were imaged using an Olympus FluoView 3000 confocal microscope.

\section{Bacterial strain and fly injection}

The Streptococcus pneumoniae strain EJ1 was stored at $-80^{\circ} \mathrm{C}$ in Tryptic Soy Broth (TSB) media containing $10 \%$ glycerol. For the experiments, bacteria were streaked onto agar plates containing $3 \%$ TSB and $100 \mathrm{mg} / \mathrm{mL}$ streptomycin and subsequently incubated at $37^{\circ} \mathrm{C}$ in $5 \% \mathrm{CO}_{2}$ overnight. Single colonies were inoculated into $3 \mathrm{~mL}$ of TSB liquid media with $100 \mathrm{mg} / \mathrm{mL}$ of streptomycin and 100,000 units of catalase and incubated at $37^{\circ} \mathrm{C}+5 \% \mathrm{CO} 2$ overnight. Bacterial density was measured after an additional $4 \mathrm{~h}$ so that it reached an approximate 0.4 OD600. Final bacterial cultures were centrifuged and dissolved in PBS so the final OD reached 2.4. The S. pneumoniae culture was kept on ice prior to injection and during the injection itself. Seven-day-old males were anaesthetized with $\mathrm{CO}_{2}$ and injected with $50 \mathrm{~nL}$ culture containing 20,000 S. pneumoniae bacteria or $50 \mathrm{~nL}$ of mock buffer (PBS) into the ventrolateral side of the abdomen using an Eppendorf Femtojet microinjector. The Listeria monocytogenes strain $10403 \mathrm{~S}$ was stored at $-80^{\circ} \mathrm{C}$ in brain and heart infusion (BHI) broth containing $25 \%$ glycerol. For the experiments bacteria were streaked onto Luria Bertani (LB) agar plates containing $100 \mu \mathrm{g} / \mathrm{mL}$ streptomycin and incubated at $37^{\circ} \mathrm{C}$ overnight; plates were stored at $4^{\circ} \mathrm{C}$ and used for inoculation for a period of two weeks. Single colonies were used to inoculate $3 \mathrm{~mL}$ of $\mathrm{BHI}$ and incubated overnight at $37^{\circ} \mathrm{C}$ without shaking to obtain a morning $600 \mathrm{~nm}$ optical density (OD600) of approx. 0.4. Further, L. monocytogenes cultures were diluted to OD600 0.01 in phosphate buffered saline (PBS) and stored on ice prior to loading into an injection needle. Approximately 1,000 Listeria per fly were injected.

\section{Survival analysis}

Streptococcus-injected flies were kept at $29^{\circ} \mathrm{C}$ in vials with approximately 30 individuals per vial and were transferred to fresh food every other day. Listeria-injected flies were kept at $25^{\circ} \mathrm{C}$. Dead flies were counted daily. At least three independent experiments were performed and combined into a single survival curve generated in Graphpad Prism software; individual experiments showed comparable results. The average number of individuals was more than 500 for each genotype and replicate. Data were analyzed by Log-rank and Grehan-Breslow Wilcoxon tests (which gave more weight to deaths at early time points). 
621

622

623

624

625

626

627

628

629

630

631

632

633

634

635

636

637

638

639

640

641

642

643

644

\section{Pathogen load measurement}

Single flies were homogenized in PBS using a motorized plastic pestle in $1.5 \mathrm{~mL}$ tubes. Bacteria were plated in spots onto LB (L. monocytogenes) or TSB (S. pneumoniae) agar plates containing streptomycin in serial dilutions and incubated overnight at $37^{\circ} \mathrm{C}$ before manual counting. To determine intracellular L. monocytogenes loads, flies were injected with $50 \mathrm{~nL}$ of gentamycin solution $(1 \mathrm{mg} / \mathrm{mL}$ in PBS) $3 \mathrm{~h}$ prior to fly homogenization. Pathogen loads of 16 flies were determined for each genotype and treatment in each experiment; at least three independent infection experiments were conducted and the results were combined into one graph (in all presented cases, individual experiments showed comparable results).

\section{THP-1 cell lines}

THP-1 cells were cultured at $37^{\circ} \mathrm{C}, 5 \% \mathrm{CO} 2$, in RPMI-1640 medium (Sigma), supplemented with $2 \mathrm{mM}$ glutamine (Applichem), $2 \mathrm{~g} / \mathrm{L}$ sodium bicarbonate (J\&K Scientific), 10\% FBS (Biosera) and $100 \mathrm{mg} / \mathrm{L}$ streptomycin (Sigma). Prior to the experiment, cells were transferred to 24 -well plates at $10^{5}$ cells/well in four biological replicates. THP-1 cells were activated with phorbol-12-myristate-13acetate $(200 \mathrm{ng} / \mathrm{mL}$, MedChemExpress). After 24 hours, S. pneumoniae bacteria were added (MOI 50 bacteria/macrophage) and the plate was centrifuged briefly ( $2 \mathrm{~min}, 200 \times \mathrm{g}$ ). Following $6-\mathrm{h}$ incubation, the cells were washed with RPMI-1640 medium, and fresh RPMI-1640 supplemented with gentamycin $(0.1 \mathrm{mg} / \mathrm{mL}$, Sigma) was added. After $1 \mathrm{~h}$ incubation, the medium was replaced with RPMI-1640 supplemented with penicillin-streptomycin (1\%, Biosera). After amother $17 \mathrm{~h}$, the cells were harvested into TRIzol Reagent (Invitrogen) followed by RNA isolation.

\section{Key resources table}

\begin{tabular}{|c|c|c|c|c|}
\hline $\begin{array}{l}\text { Reagent type } \\
\text { (species) or } \\
\text { resource }\end{array}$ & Designation & $\begin{array}{l}\text { Source } \\
\text { reference }\end{array}$ & Identifier & $\begin{array}{l}\text { Additional } \\
\text { information }\end{array}$ \\
\hline $\begin{array}{l}\text { Strain, strain } \\
\text { background } \\
\text { (Streptococcus } \\
\text { pneumoniae) }\end{array}$ & EJ1 strain & $\begin{array}{l}\text { Provided by David } \\
\text { Schneider }\end{array}$ & & \begin{tabular}{|l} 
Dilution \\
20,000 units
\end{tabular} \\
\hline $\begin{array}{l}\text { Chemical } \\
\text { compound, } \\
\text { drug }\end{array}$ & TRIzol Reagent & Invitrogen & $\begin{array}{l}\text { Cat\# 15- } \\
596-018\end{array}$ & \\
\hline $\begin{array}{l}\text { Chemical } \\
\text { compound, } \\
\text { drug }\end{array}$ & $\begin{array}{l}\text { Superscript III Reverseerse } \\
\text { Transcriptase }\end{array}$ & Invitrogen & $\begin{array}{l}\text { Cat\# } \\
18080044\end{array}$ & \\
\hline $\begin{array}{l}\text { Chemical } \\
\text { compound, } \\
\text { drug }\end{array}$ & 2x SYBR Master Mix & Top-Bio & Cat\# T607 & \\
\hline
\end{tabular}




\begin{tabular}{|c|c|c|c|c|}
\hline $\begin{array}{l}\text { Reagent type } \\
\text { (species) or } \\
\text { resource }\end{array}$ & Designation & $\begin{array}{c}\text { Source } \\
\text { reference }\end{array}$ & Identifier & $\begin{array}{l}\text { Additional } \\
\text { information }\end{array}$ \\
\hline $\begin{array}{l}\text { Chemical } \\
\text { compound, } \\
\text { drug }\end{array}$ & Grace's Insect Medium & Sigma & $\begin{array}{l}\text { Cat\# } \\
\text { G9771-1L }\end{array}$ & \\
\hline $\begin{array}{l}\text { Chemical } \\
\text { compound, } \\
\text { drug }\end{array}$ & Oil Red O & Sigma & \begin{tabular}{|l|} 
Cat\# \\
O0625-25G
\end{tabular} & \\
\hline $\begin{array}{l}\text { Chemical } \\
\text { compound, } \\
\text { drug }\end{array}$ & DAPI & Sigma & $\begin{array}{l}\text { Cat\# } \\
\text { D9542 }\end{array}$ & \\
\hline $\begin{array}{l}\text { Chemical } \\
\text { compound, } \\
\text { drug }\end{array}$ & $\begin{array}{l}\text { Cell Brite Fix Membrane Stain } \\
488\end{array}$ & Biotium & $\begin{array}{l}\text { Cat\# } \\
30090-\mathrm{T}\end{array}$ & \\
\hline $\begin{array}{l}\text { Chemical } \\
\text { compound, } \\
\text { drug }\end{array}$ & Phalloidin blue & ThermoFisher & $\begin{array}{l}\text { Cat\# } \\
\text { A30104 }\end{array}$ & \\
\hline $\begin{array}{l}\text { Chemical } \\
\text { compound, } \\
\text { drug }\end{array}$ & $\begin{array}{l}\text { pHrodo }^{\text {TM }} \text { Red S. aureus } \\
\text { Bioparticles }{ }^{\mathrm{TM}} \text { Conjugate for } \\
\text { Phagocytosis }\end{array}$ & ThermoFisher & \begin{tabular}{|l|} 
Cat\# \\
A10010
\end{tabular} & \\
\hline $\begin{array}{l}\text { Chemical } \\
\text { compound, } \\
\text { drug }\end{array}$ & $\begin{array}{l}\text { Phorbol-12-myristate-13- } \\
\text { acetate }\end{array}$ & MedChemExpress & $\begin{array}{l}\text { Cat\# } \\
\text { HY-18739 }\end{array}$ & \\
\hline $\begin{array}{l}\text { Chemical } \\
\text { compound, } \\
\text { drug }\end{array}$ & RPMI-1640 Medium & Sigma & $\begin{array}{l}\text { Cat\# } \\
\text { R7755 }\end{array}$ & \\
\hline $\begin{array}{l}\text { Chemical } \\
\text { compound, } \\
\text { drug }\end{array}$ & Glutamine & Applichem & $\begin{array}{l}\text { Cat\# } \\
\text { A3704 }\end{array}$ & \\
\hline $\begin{array}{l}\text { Chemical } \\
\text { compound, } \\
\text { drug }\end{array}$ & Gentamycin & Sigma & $\begin{array}{l}\text { Cat\# } \\
1405-41-0\end{array}$ & \\
\hline $\begin{array}{l}\text { Chemical } \\
\text { compound, } \\
\text { drug }\end{array}$ & FBS & Biosera & \begin{tabular}{|l} 
Cat\# \\
FB-1001
\end{tabular} & \\
\hline Chemical & Penicilin-Streptomycin & Biosera & Cat\# & \\
\hline
\end{tabular}




\begin{tabular}{|c|c|c|c|c|}
\hline $\begin{array}{l}\text { Reagent type } \\
\text { (species) or } \\
\text { resource }\end{array}$ & Designation & $\begin{array}{l}\text { Source } \\
\text { reference }\end{array}$ & Identifier & $\begin{array}{l}\text { Additional } \\
\text { information }\end{array}$ \\
\hline $\begin{array}{l}\text { compound, } \\
\text { drug }\end{array}$ & & & 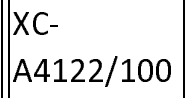 & \\
\hline $\begin{array}{l}\text { Chemical } \\
\text { compound, } \\
\text { drug }\end{array}$ & Protease inhibitor mixture & Sigma & Cat\# & \\
\hline $\begin{array}{l}\text { Chemical } \\
\text { compound, } \\
\text { drug }\end{array}$ & Phosphatase inhibitor & Thermo-Fisher & Cat\# & \\
\hline Antibody & anti-Akt & Cell Signaling Technology & Cat\# & \\
\hline Antibody & anti-phospho Ser505-Akt & Cell Signaling Technology & Cat\# & \\
\hline Antibody & Alpha-Catenin & $\begin{array}{l}\text { Development Studies } \\
\text { Hybridoma Bank }\end{array}$ & $\begin{array}{l}\text { Cat\# } \\
\text { DCAT-1 }\end{array}$ & \\
\hline $\begin{array}{l}\text { Chemical } \\
\text { compound, } \\
\text { drug }\end{array}$ & SuperSignal ECL substrate & Thermo-Fisher & $\begin{array}{l}\text { Cat\# } \\
34075\end{array}$ & \\
\hline Antibody & Rabbit Drosophila anti-Foxo & CosmoBio & $\begin{array}{l}\text { Cat\# THU- } \\
\text { A-DFoxo }\end{array}$ & \\
\hline Antibody & Cy2 Goat anti-Rabbit & \begin{tabular}{|l|} 
Jackson- \\
Immunoresearch
\end{tabular} & $\begin{array}{l}\mathrm{AB} \_233802 \\
1\end{array}$ & \\
\hline $\begin{array}{l}\text { Commercial } \\
\text { assay, kit }\end{array}$ & Glucose (GO) Assay Kit & Sigma & $\begin{array}{l}\text { Cat\# } \\
\text { GAGO20- } \\
1 \mathrm{KT}\end{array}$ & \\
\hline $\begin{array}{l}\text { Commercial } \\
\text { assay, kit }\end{array}$ & Bicinchoninic Acid Assay Kit & Sigma & Cat\# BCA1 & \\
\hline $\begin{array}{l}\text { Commercial } \\
\text { assay, kit }\end{array}$ & $\begin{array}{l}\text { Cholesterol/Cholesteryl Ester } \\
\text { Quantitation Kit }\end{array}$ & Sigma & \begin{tabular}{|l} 
Cat\# \\
$428901-$ \\
$1 \mathrm{KIT}$
\end{tabular} & \\
\hline $\begin{array}{l}\text { Commercial } \\
\text { assay, kit }\end{array}$ & $\begin{array}{l}\text { Triglyceride quantification } \\
\text { Colorimetric/Fluorometric Kit }\end{array}$ & Sigma & \begin{tabular}{|l} 
Cat\# \\
MAK266-
\end{tabular} & \\
\hline
\end{tabular}




\begin{tabular}{|c|c|c|c|c|}
\hline $\begin{array}{l}\text { Reagent type } \\
\text { (species) or } \\
\text { resource }\end{array}$ & Designation & $\begin{array}{l}\text { Source } \\
\text { reference }\end{array}$ & Identifier & $\begin{array}{l}\text { Additional } \\
\text { information }\end{array}$ \\
\hline & & & $1 \mathrm{KT}$ & \\
\hline $\begin{array}{l}\text { Chemical } \\
\text { compound, } \\
\text { drug }\end{array}$ & Trehalase from porcine kidney & Sigma & \begin{tabular}{|l|} 
Cat\# \\
T8778-1UN
\end{tabular} & \\
\hline $\begin{array}{l}\text { Genetic reagent } \\
\text { (Drosophila } \\
\text { melanogaster) }\end{array}$ & 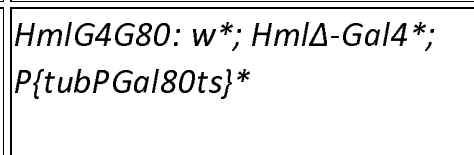 & $\begin{array}{l}\text { Cross made in our } \\
\text { laboratory by Tomas } \\
\text { Dolezal }\end{array}$ & & \\
\hline $\begin{array}{l}\text { Genetic reagent } \\
\text { (Drosophila } \\
\text { melanogaster) }\end{array}$ & $\operatorname{ImpL2}{ }^{\text {RNAi }}$ & \begin{tabular}{|l} 
Bloomington \\
Drosophila Stock Center
\end{tabular} & & \\
\hline $\begin{array}{l}\text { Genetic reagent } \\
\text { (Drosophila } \\
\text { melanogaster) }\end{array}$ & ImpL2 Gal4 & $\begin{array}{l}\text { Provided by Hugo } \\
\text { Stocker }\end{array}$ & & \\
\hline $\begin{array}{l}\text { Genetic reagent } \\
\text { (Drosophila } \\
\text { melanogaster) }\end{array}$ & Atg8a-mCherry & $\begin{array}{l}\text { Provided by Gabor } \\
\text { Juhasz }\end{array}$ & & \\
\hline $\begin{array}{l}\text { Genetic reagent } \\
\text { (Drosophila } \\
\text { melanogaster) }\end{array}$ & ImpL2 ${ }^{\text {cds }}$ & $\begin{array}{l}\text { Provided by Hugo } \\
\text { Stocker }\end{array}$ & & \\
\hline $\begin{array}{l}\text { Genetic reagent } \\
\text { (Drosophila } \\
\text { melanogaster) }\end{array}$ & 20xUAS-6xmCherry & \begin{tabular}{|l} 
Bloomington \\
Drosophila Stock Center
\end{tabular} & & \\
\hline $\begin{array}{l}\text { Genetic reagent } \\
\text { (Drosophila } \\
\text { melanogaster) }\end{array}$ & CrqGal4>2xeGFP & $\begin{array}{l}\text { Provided by Marc C. } \\
\text { Dionne }\end{array}$ & & \\
\hline $\begin{array}{l}\text { Genetic reagent } \\
(D . \\
\text { melanogaster) }\end{array}$ & 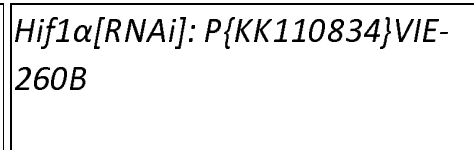 & $\begin{array}{l}\text { Vienna Drosophila } \\
\text { Resource Center }\end{array}$ & \begin{tabular}{|l|} 
VDRC: \\
v106504
\end{tabular} & FBst0478328 \\
\hline $\begin{array}{l}\text { Genetic reagent } \\
\text { (D. melanogaste } \\
r \text { ) }\end{array}$ & $\begin{array}{l}\text { TRiP }{ }^{\text {control }}: y(1) v(1) \\
P\{y[+t 7.7]=\text { CaryP }\} a t t P 2\end{array}$ & $\begin{array}{l}\text { Bloomington } \\
\text { Drosophila Stock Center }\end{array}$ & $\begin{array}{l}\text { BDSC: } \\
36303\end{array}$ & FBst0036303 \\
\hline $\begin{array}{l}\text { Genetic reagent } \\
(D . \\
\text { melanogaster) }\end{array}$ & $w: w^{1118}$ & $\begin{array}{l}\text { Genetic background } \\
\text { based on Cantons }\end{array}$ & & \\
\hline
\end{tabular}




\begin{tabular}{|c|c|c|c|c|}
\hline $\begin{array}{l}\text { Reagent type } \\
\text { (species) or } \\
\text { resource }\end{array}$ & Designation & $\begin{array}{c}\text { Source } \\
\text { reference }\end{array}$ & Identifier & $\begin{array}{l}\text { Additional } \\
\text { information }\end{array}$ \\
\hline $\begin{array}{l}\text { Sequence-based } \\
\text { reagent }\end{array}$ & $\begin{array}{l}\text { Foxo forward: } \\
\text { CAATGCAAGAGATGGTCTCTC }\end{array}$ & KRD & CG3143 & FBgn0038197 \\
\hline $\begin{array}{l}\text { Sequence-based } \\
\text { reagent }\end{array}$ & $\begin{array}{l}\text { Foxo reverse: } \\
\text { TGACCAGGTTCGTGTTGACC }\end{array}$ & KRD & CG3143 & FBgn0038197 \\
\hline $\begin{array}{l}\text { Sequence-based } \\
\text { reagent }\end{array}$ & $\begin{array}{l}\text { 4EBP Forward: } \\
\text { CCATGATCACCAGGAAGGTTGTC } \\
\text { A }\end{array}$ & KRD & CG8846 & FBgn0261560 \\
\hline $\begin{array}{l}\text { Sequence-based } \\
\text { reagent }\end{array}$ & $\begin{array}{l}\text { 4EBP Reverse: } \\
\text { AGCCCGCTCGTAGATAAGTTTGG } \\
\mathrm{T}\end{array}$ & KRD & CG8846 & FBgn0261560 \\
\hline \begin{tabular}{|l|} 
Sequence-based \\
reagent
\end{tabular} & $\begin{array}{l}\text { EIF-4E Forward: } \\
\text { AAGAAGAACATTCGCCCATG T }\end{array}$ & KRD & CG4035 & FBgn0015218 \\
\hline $\begin{array}{l}\text { Sequence-based } \\
\text { reagent }\end{array}$ & $\begin{array}{l}\text { EIF-4E Reverse: } \\
\text { GGACTGCCGACGGAAACAAC }\end{array}$ & KRD & CG4035 & FBgn0015218 \\
\hline \begin{tabular}{|l|} 
Sequence-based \\
reagent
\end{tabular} & $\begin{array}{l}\text { Glut-CoA deh Forward: } \\
\text { CTGATTGGTGCCTTGGCC }\end{array}$ & KRD & CG9547 & FBgn0031824 \\
\hline $\begin{array}{l}\text { Sequence-based } \\
\text { reagent }\end{array}$ & $\begin{array}{l}\text { Glut-CoA deh Reverse: } \\
\text { ATAGCGAGCGTTGTTCAGAC }\end{array}$ & KRD & CG9547 & FBgn0031824 \\
\hline \begin{tabular}{|l|} 
Sequence-based \\
reagent
\end{tabular} & $\begin{array}{l}\text { Lip4 Forward: } \\
\text { CCACTTGGGTGATGATGGGAC }\end{array}$ & KRD & CG6113 & FBgn0032264 \\
\hline \begin{tabular}{|l|} 
Sequence-based \\
reagent
\end{tabular} & $\begin{array}{l}\text { Lip4 Reverse: } \\
\text { ACGTATTGCCACGAACATTGG }\end{array}$ & KRD & CG6113 & FBgn0032264 \\
\hline \begin{tabular}{|l|} 
Sequence-based \\
reagent
\end{tabular} & $\begin{array}{l}\text { MTP Forward: } \\
\text { GAATCGAAATGCCCAGACG }\end{array}$ & KRD & CG9342 & FBgn0266369 \\
\hline \begin{tabular}{|l|} 
Sequence-based \\
reagent
\end{tabular} & $\begin{array}{l}\text { MTP Reverse: } \\
\text { AACGTTGGTTGTTTGAGAAGC }\end{array}$ & KRD & CG9342 & FBgn0266369 \\
\hline \begin{tabular}{|l|} 
Sequence-based \\
reagent
\end{tabular} & $\begin{array}{l}\text { HSL Forward: } \\
\text { CAGTCCTACGAGATTCACGG }\end{array}$ & KRD & CG11055 & FBgn0034491 \\
\hline \begin{tabular}{|l|} 
Sequence-based \\
reagent
\end{tabular} & $\begin{array}{l}\text { HSL Reverse: } \\
\text { GGCTTCGTTGGATAACATTGTG }\end{array}$ & KRD & CG11055 & FBgn0034491 \\
\hline Sequence-based & $\begin{array}{l}\text { Bmm Forward: } \\
\text { CACCGCGCCGCAATGAATGTATA }\end{array}$ & KRD & CG5295 & FBgn0036449 \\
\hline
\end{tabular}




\begin{tabular}{|c|c|c|c|c|}
\hline $\begin{array}{l}\text { Reagent type } \\
\text { (species) or } \\
\text { resource }\end{array}$ & Designation & $\begin{array}{l}\text { Source } \\
\text { reference }\end{array}$ & Identifier & $\begin{array}{l}\text { Additional } \\
\text { information }\end{array}$ \\
\hline reagent & A & & & \\
\hline \begin{tabular}{|l|} 
Sequence-based \\
reagent
\end{tabular} & $\begin{array}{l}\text { Bmm Reverse: } \\
\text { TTCAATCACTGTTTGTCGGTCGG } \\
\text { C }\end{array}$ & KRD & CG5295 & FBgn0036449 \\
\hline \begin{tabular}{|l|} 
Sequence-based \\
reagent
\end{tabular} & $\begin{array}{l}\text { GlyP Forward: } \\
\text { TCCACCCTGAGGGACTACTAC }\end{array}$ & KRD & CG7254 & FBgn0004507 \\
\hline \begin{tabular}{|l|} 
Sequence-based \\
reagent
\end{tabular} & $\begin{array}{l}\text { GlyP Reverse: } \\
\text { GGTGTTGGTCAGTGAGCGAC }\end{array}$ & KRD & CG7254 & FBgn0004507 \\
\hline \begin{tabular}{l|} 
Sequence-based \\
reagent
\end{tabular} & $\begin{array}{l}\text { GlyS Forward: } \\
\text { CGATGAAGAAGTACAGGGTC }\end{array}$ & KRD & CG6904 & FBgn0266064 \\
\hline \begin{tabular}{l|} 
Sequence-based \\
reagent
\end{tabular} & $\begin{array}{l}\text { GlyS Reverse: } \\
\text { TATCATCGTTATTGCCTGGAG }\end{array}$ & KRD & CG6904 & FBgn0266064 \\
\hline $\begin{array}{l}\text { Sequence-based } \\
\text { reagent }\end{array}$ & $\begin{array}{l}\text { apoLTP Forward: } \\
\text { TGGGTGGATTGAAGCCACAG }\end{array}$ & KRD & CG15828 & FBgn0032136 \\
\hline \begin{tabular}{|l|} 
Sequence-based \\
reagent
\end{tabular} & $\begin{array}{l}\text { apoLTP Reverse: } \\
\text { TGAGTGGATTTCTCTCACTGC }\end{array}$ & KRD & CG15828 & FBgn0032136 \\
\hline \begin{tabular}{|l|} 
Sequence-based \\
reagent
\end{tabular} & $\begin{array}{l}\text { apoLPP Forward: } \\
\text { TTGGAATCCTAGCTTCTGTGCT }\end{array}$ & KRD & CG11064 & FBgn0087002 \\
\hline \begin{tabular}{|l|} 
Sequence-based \\
reagent
\end{tabular} & $\begin{array}{l}\text { apoLPP Reverse: } \\
\text { AGTCATAGTAGTTGCCGGGTAT }\end{array}$ & KRD & CG11064 & FBgn0087002 \\
\hline \begin{tabular}{|l|} 
Sequence-based \\
reagent
\end{tabular} & $\begin{array}{l}\text { Atg1 Forward: } \\
\text { CTAAAGCCGTCGTCCAATGT }\end{array}$ & KRD & CG10967 & FBgn0260945 \\
\hline \begin{tabular}{|l|} 
Sequence-based \\
reagent
\end{tabular} & $\begin{array}{l}\text { Atg1 Reverse: } \\
\text { GAACAGCATGCTCCGGTATT }\end{array}$ & KRD & CG10967 & FBgn0260945 \\
\hline \begin{tabular}{|l|} 
Sequence-based \\
reagent
\end{tabular} & $\begin{array}{l}\text { Atg6 Forward: } \\
\text { CGGAGTTATCTTTGCCATCT }\end{array}$ & KRD & CG5429 & FBgn0264325 \\
\hline \begin{tabular}{|l|} 
Sequence-based \\
reagent
\end{tabular} & $\begin{array}{l}\text { Atg6 Reverse: } \\
\text { GTGCTACACGCTGTTGCTC }\end{array}$ & KRD & CG5429 & FBgn0264325 \\
\hline $\begin{array}{l}\text { Sequence-based } \\
\text { reagent }\end{array}$ & $\begin{array}{l}\text { Rp49 Forward: } \\
\text { AAGCTGTCGCACAAATGGCG }\end{array}$ & KRD & CG7939 & FBgn0002626 \\
\hline
\end{tabular}




\begin{tabular}{|c|c|c|c|c|}
\hline $\begin{array}{l}\text { Reagent type } \\
\text { (species) or } \\
\text { resource }\end{array}$ & Designation & $\begin{array}{l}\text { Source } \\
\text { reference }\end{array}$ & Identifier & $\begin{array}{l}\text { Additional } \\
\text { information }\end{array}$ \\
\hline $\begin{array}{l}\text { Sequence-based } \\
\text { reagent }\end{array}$ & $\begin{array}{l}\text { Rp49 Reverse: } \\
\text { GCACGTTGTGCACCAGGAAC }\end{array}$ & KRD & CG7939 & FBgn0002626 \\
\hline $\begin{array}{l}\text { Sequence-based } \\
\text { reagent }\end{array}$ & $\begin{array}{l}\text { ImpL2 Forward: } \\
\text { TTCGCGGTTTCTGGGCACCC }\end{array}$ & KRD & CG15009 & FBgn0001257 \\
\hline $\begin{array}{l}\text { Sequence-based } \\
\text { reagent }\end{array}$ & $\begin{array}{l}\text { ImpL2 Reverse: } \\
\text { GCGCGTCCGATCGTCGCATA }\end{array}$ & KRD & CG15009 & FBgn0001257 \\
\hline $\begin{array}{l}\text { Sequence-based } \\
\text { reagent }\end{array}$ & $\begin{array}{l}\text { ImpL2-RA Forward: } \\
\text { GTGCCAACGAAGCTTCGAGTG }\end{array}$ & KRD & CG15009 & FBgn0001257 \\
\hline $\begin{array}{l}\text { Sequence-based } \\
\text { reagent }\end{array}$ & $\begin{array}{l}\text { ImpL2-RA Reverse: } \\
\text { GCGTGGCTTCTCCTCCTCC }\end{array}$ & KRD & CG15009 & FBgn0001257 \\
\hline $\begin{array}{l}\text { Sequence-based } \\
\text { reagent }\end{array}$ & $\begin{array}{l}\text { ImpL2-RB, D Forward: } \\
\text { GTCGTCGGAAAGGATACCGC }\end{array}$ & KRD & CG15009 & FBgn0001257 \\
\hline $\begin{array}{l}\text { Sequence-based } \\
\text { reagent }\end{array}$ & $\begin{array}{l}\text { ImpL2-RB, D Reverse: } \\
\text { TTCCGTCGTCAATCCAATAGCC }\end{array}$ & KRD & CG15009 & FBgn0001257 \\
\hline \begin{tabular}{l|} 
Sequence-based \\
reagent
\end{tabular} & $\begin{array}{l}\text { ImpL2-RC Forward: } \\
\text { ATAGACTCGGAGACCTCCCC }\end{array}$ & KRD & CG15009 & FBgn0001257 \\
\hline \begin{tabular}{l|} 
Sequence-based \\
reagent
\end{tabular} & $\begin{array}{l}\text { ImpL2-RC Reverse: } \\
\text { GCGTGGCTTCTCCTCCTCC }\end{array}$ & KRD & CG15009 & FBgn0001257 \\
\hline \begin{tabular}{l|} 
Sequence-based \\
reagent
\end{tabular} & $\begin{array}{l}\text { IGFBP7 Forward: } \\
\text { GCCATCACCCAGGTCAGCAAG }\end{array}$ & KRD & $\begin{array}{l}\text { GC04M057 } \\
030\end{array}$ & \\
\hline $\begin{array}{l}\text { Sequence-based } \\
\text { reagent }\end{array}$ & $\begin{array}{l}\text { IGFBP7 Reverse: } \\
\text { GGATTCCGATGACCTCACAGCT }\end{array}$ & KRD & $\begin{array}{l}\text { GC04M057 } \\
030\end{array}$ & \\
\hline \begin{tabular}{l|} 
Sequence-based \\
reagent
\end{tabular} & $\begin{array}{l}\text { ACTB Forward: } \\
\text { ATTGCCGACAGGATGCAGAA }\end{array}$ & KRD & $\begin{array}{l}\text { GC07M005 } \\
527\end{array}$ & \\
\hline \begin{tabular}{|l||} 
Sequence-based \\
reagent
\end{tabular} & $\begin{array}{l}\text { ACTB Reverse: } \\
\text { GCTGATCCACATCTGCTGGAA }\end{array}$ & KRD & $\begin{array}{l}\text { GC07M005 } \\
527\end{array}$ & \\
\hline
\end{tabular}




\begin{tabular}{|c|c|c|c|c|}
\hline $\begin{array}{l}\text { Reagent type } \\
\text { (species) or } \\
\text { resource }\end{array}$ & Designation & $\begin{array}{c}\text { Source } \\
\text { reference }\end{array}$ & Identifier & $\begin{array}{l}\text { Additional } \\
\text { information }\end{array}$ \\
\hline $\begin{array}{l}\text { Sequence-based } \\
\text { reagent }\end{array}$ & $\begin{array}{l}\text { IGFBP1 Forward: } \\
\text { TCCTTTGGGACGCCATCAGTAC }\end{array}$ & KRD & $\begin{array}{l}\text { GC07P046 } \\
552\end{array}$ & \\
\hline $\begin{array}{l}\text { Sequence-based } \\
\text { reagent }\end{array}$ & $\begin{array}{l}\text { IGFBP1 Reverse: } \\
\text { GATGTCTCCTGTGCCTTGGCTA }\end{array}$ & KRD & $\begin{array}{l}\text { GC07P046 } \\
552\end{array}$ & \\
\hline $\begin{array}{l}\text { Sequence-based } \\
\text { reagent }\end{array}$ & $\begin{array}{l}\text { IGFBP2 Forward: } \\
\text { CGAGGGCACTTGTGAGAAGCG }\end{array}$ & KRD & $\begin{array}{l}\text { GC02P216 } \\
632\end{array}$ & \\
\hline $\begin{array}{l}\text { Sequence-based } \\
\text { reagent }\end{array}$ & $\begin{array}{l}\text { IGFBP2 Reverse: } \\
\text { TGTTCATGGTGCTGTCCACGTG }\end{array}$ & KRD & $\begin{array}{l}\text { GC02P216 } \\
632\end{array}$ & \\
\hline $\begin{array}{l}\text { Sequence-based } \\
\text { reagent }\end{array}$ & $\begin{array}{l}\text { IGFBP3 Forward: } \\
\text { CGCTACAAAGTTGACTACGAGTC }\end{array}$ & KRD & $\begin{array}{l}\text { GC07M045 } \\
912\end{array}$ & \\
\hline $\begin{array}{l}\text { Sequence-based } \\
\text { reagent }\end{array}$ & $\begin{array}{l}\text { IGFBP3 Reverse: } \\
\text { GTCTTCCATTTCTCTACGGCAGG }\end{array}$ & KRD & $\begin{array}{l}\text { GC07M045 } \\
912\end{array}$ & \\
\hline $\begin{array}{l}\text { Sequence-based } \\
\text { reagent }\end{array}$ & $\begin{array}{l}\text { IGFBP4 Forward: } \\
\text { ACCCACGAGGACCTCTACATCA }\end{array}$ & KRD & $\begin{array}{l}\text { GC17P040 } \\
443\end{array}$ & \\
\hline $\begin{array}{l}\text { Sequence-based } \\
\text { reagent }\end{array}$ & $\begin{array}{l}\text { IGFBP4 Reverse: } \\
\text { CACACCAGCACTTGCCACGCT }\end{array}$ & KRD & $\begin{array}{l}\text { GC17P040 } \\
443\end{array}$ & \\
\hline $\begin{array}{l}\text { Sequence-based } \\
\text { reagent }\end{array}$ & $\begin{array}{l}\text { IGFBP5 Forward: } \\
\text { CGTGCTGTGTACCTGCCCAATT }\end{array}$ & KRD & $\begin{array}{l}\text { GC02M216 } \\
672\end{array}$ & \\
\hline $\begin{array}{l}\text { Sequence-based } \\
\text { reagent }\end{array}$ & $\begin{array}{l}\text { IGFBP5 Reverse: } \\
\text { ACTTGTCCACGCACCAGCAGAT }\end{array}$ & KRD & $\begin{array}{l}\text { GCO2M216 } \\
672\end{array}$ & \\
\hline $\begin{array}{l}\text { Sequence-based } \\
\text { reagent }\end{array}$ & $\begin{array}{l}\text { IGFBP6 Forward: } \\
\text { CACAGGATGTGAACCGCAGAGA }\end{array}$ & KRD & $\begin{array}{l}\text { GC12P053 } \\
097\end{array}$ & \\
\hline $\begin{array}{l}\text { Sequence-based } \\
\text { reagent }\end{array}$ & $\begin{array}{l}\text { IGFBP6 Reverse: } \\
\text { CACTGAGTCCAGATGTCTACGG }\end{array}$ & KRD & $\begin{array}{l}\text { GC12P053 } \\
097\end{array}$ & \\
\hline $\begin{array}{l}\text { Software, } \\
\text { algorithm }\end{array}$ & Graphpad Prism & $\begin{array}{l}\text { https://www.graphpad.c } \\
\text { om/ }\end{array}$ & $\begin{array}{l}\text { Graphpad } \\
\text { Prism }\end{array}$ & $\begin{array}{l}\text { RRID:SCR 00 } \\
2798\end{array}$ \\
\hline
\end{tabular}


bioRxiv preprint doi: https://doi.org/10.1101/2020.09.24.311670; this version posted September 25, 2020. The copyright holder for this preprint (which was not certified by peer review) is the author/funder, who has granted bioRxiv a license to display the preprint in perpetuity. It is made available under aCC-BY-NC-ND 4.0 International license.

\begin{tabular}{|c|c|c|c|c|}
\hline $\begin{array}{l}\text { Reagent type } \\
\text { (species) or } \\
\text { resource }\end{array}$ & Designation & $\begin{array}{l}\text { Source } \\
\text { reference }\end{array}$ & Identifier & $\begin{array}{l}\text { Additional } \\
\text { information }\end{array}$ \\
\hline $\begin{array}{l}\text { Software, } \\
\text { algorithm }\end{array}$ & Microsoft Excel & $\begin{array}{l}\text { https://www.microsoft.c } \\
\text { om/ }\end{array}$ & $\begin{array}{l}\text { Microsoft } \\
\text { Excel }\end{array}$ & \\
\hline $\begin{array}{l}\text { Software, } \\
\text { algorithm }\end{array}$ & Fiji & ImageJ - https://fiii.sc & ImageJ & $\begin{array}{l}\text { RRID:SCR 00 } \\
2 \underline{285}\end{array}$ \\
\hline Other & S3e Cell Sorter & $\begin{array}{l}\text { BioRad -http://www.bio- } \\
\text { rad.com/ }\end{array}$ & BioRad & \\
\hline Other & Olympus FluoView 3000 & $\begin{array}{l}\text { Olympus - } \\
\text { https://www.olympus- } \\
\text { global.com/ }\end{array}$ & Olympus & $\begin{array}{l}\text { RRID:SCR } 01 \\
\begin{array}{ll}7015 \\
\text { RRID:SCR } & 01 \\
4215\end{array} \\
\end{array}$ \\
\hline Other & Olympus IX71 & $\begin{array}{l}\text { Olympus - } \\
\text { https://www.olympus- } \\
\text { global.com/ }\end{array}$ & Olympus & \\
\hline
\end{tabular}

645

646 
647

648

649

650

651

652

653

654

655

656

657

658

659

660

661

662

663

664

665

666

667

668

669

670

671

672

673

674

675

676

677

678

679

680

681

682

683

684

685

686

687

688

689

\section{References}

Alee. 2011. "ImpL2 Represses Insulin Signaling in Response to Hypoxia." Dissertation Thesis. University of Oregon, Eugene, Oregon, USA.

Akiel, Maaged et al. 2017. "IGFBP7 Deletion Promotes Hepatocellular Carcinoma." Cancer Research 77(15): 4014-25. http://cancerres. aacrjournals. org/lookup/doi/10.1158/0008-5472.CAN-162885.

Alic, Nazif, Matthew P. Hoddinott, Giovanna Vinti, and Linda Partridge. 2011. "Lifespan Extension by Increased Expression of the Drosophila Homologue of the IGFBP7 Tumour Suppressor." Aging Cell 10(1): 137-47. http://doi.wiley.com/10.1111/j.1474-9726.2010.00653.x.

Andrejeva, Gabriela, and Jeffrey C. Rathmell. 2017. "Similarities and Distinctions of Cancer and Immune Metabolism in Inflammation and Tumors." Cell Metabolism 26(1): 49-70. https://linkinghub.elsevier.com/retrieve/pii/S1550413117303467.

Arquier, Nathalie et al. 2006. "Analysis of the Hypoxia-Sensing Pathway in Drosophila Melanogaster." Biochemical Journal 393(2): 471-80.

https://portlandpress.com/biochemj/article/393/2/471/78893/Analysis-of-the-hypoxiasensingpathway-in.

Aspichueta, Patricia et al. 2012. "Disrupted VLDL Features and Lipoprotein Metabolism in Sepsis." In Dyslipidemia - From Prevention to Treatment, InTech.

http://www.intechopen.com/books/dyslipidemia-from-prevention-to-treatment/disruptedvldl-features-and-lipoprotein-metabolism-in-sepsis.

Bader, R. et al. 2013. "The IGFBP7 Homolog Imp-L2 Promotes Insulin Signaling in Distinct Neurons of the Drosophila Brain." Journal of Cell Science 126(12): 2571-76. http://jcs. biologists.org/cgi/doi/10.1242/jcs.120261.

Bajgar, Adam et al. 2015. "Extracellular Adenosine Mediates a Systemic Metabolic Switch during Immune Response" ed. Marc S. Dionne. PLOS Biology 13(4): e1002135. https://dx.plos.org/10.1371/journal.pbio.1002135.

Bajgar, Adam, and Tomas Dolezal. 2018. "Extracellular Adenosine Modulates Host-Pathogen Interactions through Regulation of Systemic Metabolism during Immune Response in Drosophila" ed. Brian P. Lazzaro. PLOS Pathogens 14(4): e1007022. https://dx.plos.org/10.1371/journal.ppat.1007022.

Bayley, Jeppe Seamus et al. 2020. "Cold-Acclimation Increases Depolarization Resistance and Tolerance in Muscle Fibers from a Chill-Susceptible Insect, Locusta Migratoria." American Journal of Physiology-Regulatory, Integrative and Comparative Physiology: ajpregu.00068.2020. https://journals. physiology.org/doi/10.1152/ajpregu.00068.2020.

Biswas, Subhra K., and Alberto Mantovani. 2012. "Orchestration of Metabolism by Macrophages." Cell Metabolism 15(4): 432-37. https://linkinghub.elsevier.com/retrieve/pii/S1550413112000150.

Van den Bossche, Jan, Luke A. O'Neill, and Deepthi Menon. 2017. "Macrophage Immunometabolism: Where Are We (Going)?" Trends in Immunology 38(6): 395-406. https://linkinghub.elsevier.com/retrieve/pii/S147149061730042X.

Bunker, Brandon D et al. 2015. "The Transcriptional Response to Tumorigenic Polarity Loss in Drosophila." elife 4. http://www.ncbi.nlm.nih.gov/pubmed/25719210.

Chistiakov, Dimitry A. et al. 2017. "Mechanisms of Foam Cell Formation in Atherosclerosis." Journal 
of Molecular Medicine 95(11): 1153-65. http://link.springer.com/10.1007/s00109-017-1575-8.

691

692

693

694

695

696

697

698

699

700

701

702

703

704

705

706

707

708

709

710

711

712

713

714

715

716

717

718

719

720

721

722

723

724

725

726

727

728

729

730

731

732

Dev, R, E. Bruera, and S. Dalal. 2018. "Insulin Resistance and Body Composition in Cancer Patients." Annals of Oncology 29: ii18-ii26. https://linkinghub.elsevier.com/retrieve/pii/S0923753419316801.

Dionne, Marc S., Linh N. Pham, Mimi Shirasu-Hiza, and David S. Schneider. 2006. "Akt and Foxo Dysregulation Contribute to Infection-Induced Wasting in Drosophila." Current Biology 16(20): 1977-85. https://linkinghub.elsevier.com/retrieve/pii/S0960982206020641.

Dolezal, Tomas et al. 2019. "Molecular Regulations of Metabolism during Immune Response in Insects." Insect Biochemistry and Molecular Biology 109: 31-42. https://linkinghub.elsevier.com/retrieve/pii/S0965174818304600.

Febbraio, Maria, Ella Guy, and Roy L. Silverstein. 2004. "Stem Cell Transplantation Reveals That Absence of Macrophage CD36 Is Protective Against Atherosclerosis." Arteriosclerosis, Thrombosis, and Vascular Biology 24(12): 2333-38. https://www.ahajournals.org/doi/10.1161/01.ATV.0000148007.06370.68.

Figueroa-Clarevega, Alejandra, and David Bilder. 2015. "Malignant Drosophila Tumors Interrupt Insulin Signaling to Induce Cachexia-like Wasting." Developmental Cell 33(1): 47-55. https://linkinghub.elsevier.com/retrieve/pii/S1534580715001434.

Gunnerson, Kyle J. et al. 2016. "TIMP2•IGFBP7 Biomarker Panel Accurately Predicts Acute Kidney Injury in High-Risk Surgical Patients." Journal of Trauma and Acute Care Surgery 80(2): 243-49. http://journals.Iww.com/01586154-201602000-00009.

H. Tomkin, Gerald. 2012. "LDL as a Cause of Atherosclerosis." The Open Atherosclerosis \& Thrombosis Journal 5(1): 13-21. http://benthamopen.com/ABSTRACT/TOATHERTJ-5-13.

Harris, H W, J E Gosnell, and Z L Kumwenda. 2000. "The Lipemia of Sepsis: Triglyceride-Rich Lipoproteins as Agents of Innate Immunity." Journal of endotoxin research 6(6): 421-30. http://www.ncbi.nlm.nih.gov/pubmed/11521066.

Honegger, Basil et al. 2008. "Imp-L2, a Putative Homolog of Vertebrate IGF-Binding Protein 7, Counteracts Insulin Signaling in Drosophila and Is Essential for Starvation Resistance." Journal of Biology 7(3): 10. http://jbiol.biomedcentral.com/articles/10.1186/jbiol72.

Kamagate, Adama et al. 2008. "FoxO1 Mediates Insulin-Dependent Regulation of Hepatic VLDL Production in Mice." Journal of Clinical Investigation. http://www.jci.org/articles/view/32914.

Kamps-Hughes, Nick, Jessica L. Preston, Melissa A. Randel, and Eric A. Johnson. 2015. "Genome-Wide Identification of Hypoxia-Induced Enhancer Regions." PeerJ 3: e1527. https://peerj.com/articles/1527.

Kelly, Beth, and Luke AJ O'Neill. 2015. "Metabolic Reprogramming in Macrophages and Dendritic Cells in Innate Immunity." Cell Research 25(7): 771-84. http://www.nature.com/articles/cr201568.

Khovidhunkit, Weerapan et al. 2004. "Thematic Review Series: The Pathogenesis of Atherosclerosis. Effects of Infection and Inflammation on Lipid and Lipoprotein Metabolism Mechanisms and Consequences to the Host." Journal of Lipid Research 45(7): 1169-96. http://www.jlr.org/lookup/doi/10.1194/jlr.R300019-JLR200.

Krejčová, Gabriela et al. 2019. "Drosophila Macrophages Switch to Aerobic Glycolysis to Mount Effective Antibacterial Defense." elife 8. https://elifesciences.org/articles/50414.

Kwon, Young et al. 2015. "Systemic Organ Wasting Induced by Localized Expression of the Secreted 
Insulin/IGF Antagonist ImpL2." Developmental Cell 33(1): 36-46. https://linkinghub.elsevier.com/retrieve/pii/S1534580715001148.

Li, Yan et al. 2013. "HIF- and Non-HIF-Regulated Hypoxic Responses Require the Estrogen-Related Receptor in Drosophila Melanogaster" ed. Eric Rulifson. PLoS Genetics 9(1): e1003230. https://dx.plos.org/10.1371/journal.pgen.1003230.

Liu, Yi et al. 2015. "Serum IGFBP7 Levels Associate with Insulin Resistance and the Risk of Metabolic Syndrome in a Chinese Population." Scientific Reports 5(1): 10227. http://www.nature.com/articles/srep10227.

Luong, Nancy et al. 2006. "Activated FOXO-Mediated Insulin Resistance Is Blocked by Reduction of TOR Activity." Cell Metabolism 4(2): 133-42. https://linkinghub.elsevier.com/retrieve/pii/S1550413106002348.

Martínez-Castillo, Moisés et al. 2020. "Differential Production of Insulin-like Growth Factor-Binding Proteins in Liver Fibrosis Progression." Molecular and Cellular Biochemistry 469(1-2): 65-75. http://link.springer.com/10.1007/s11010-020-03728-4.

Molaei, Maral, Crissie Vandehoef, and Jason Karpac. 2019. "NF-KB Shapes Metabolic Adaptation by Attenuating Foxo-Mediated Lipolysis in Drosophila." Developmental Cell 49(5): 802-810.e6. https://linkinghub.elsevier.com/retrieve/pii/S1534580719302795.

Morgantini, Cecilia et al. 2019. "Liver Macrophages Regulate Systemic Metabolism through NonInflammatory Factors." Nature Metabolism 1(4): 445-59. http://www.nature.com/articles/s42255-019-0044-9.

Nagao, Ayako et al. 2019. "HIF-1-Dependent Reprogramming of Glucose Metabolic Pathway of Cancer Cells and Its Therapeutic Significance." International Journal of Molecular Sciences 20(2): 238. https://www.mdpi.com/1422-0067/20/2/238.

Newsholme, P, R Curi, S Gordon, and E A Newsholme. 1986. "Metabolism of Glucose, Glutamine, Long-Chain Fatty Acids and Ketone Bodies by Murine Macrophages." Biochemical Journal 239(1): 121-25. https://portlandpress.com/biochemj/article/239/1/121/21648/Metabolism-ofglucose-glutamine-longchain-fatty.

Nicholson, David, and Lindsay B. Nicholson. 2008. "A Simple Immune System Simulation Reveals Optimal Movement and Cell Density Parameters for Successful Target Clearance." Immunology 123(4): 519-27. http://doi.wiley.com/10.1111/j.1365-2567.2007.02721.x.

Odegaard, J. I., and A. Chawla. 2013. "Pleiotropic Actions of Insulin Resistance and Inflammation in Metabolic Homeostasis." Science 339(6116): 172-77. https://www.sciencemag.org/lookup/doi/10.1126/science.1230721.

Oh, Youngman et al. 1996. "Synthesis and Characterization of Insulin-like Growth Factor-Binding Protein (IGFBP)-7." Journal of Biological Chemistry 271(48): 30322-25. http://www.jbc.org/lookup/doi/10.1074/jbc.271.48.30322.

Okamoto, N. et al. 2013. "A Secreted Decoy of InR Antagonizes Insulin/IGF Signaling to Restrict Body Growth in Drosophila." Genes \& Development 27(1): 87-97. http://genesdev.cshlp.org/cgi/doi/10.1101/gad.204479.112.

Owusu-Ansah, Edward, Wei Song, and Norbert Perrimon. 2013. "Muscle Mitohormesis Promotes Longevity via Systemic Repression of Insulin Signaling." Cell 155(3): 699-712. https://linkinghub.elsevier.com/retrieve/pii/S0092867413011586.

Péan, Claire B. et al. 2017. "Regulation of Phagocyte Triglyceride by a STAT-ATG2 Pathway Controls 
Mycobacterial Infection." Nature Communications 8(1): 14642.

http://www.nature.com/articles/ncomms14642.

Podrez, Eugene A. et al. 2000. "Macrophage Scavenger Receptor CD36 Is the Major Receptor for LDL Modified by Monocyte-Generated Reactive Nitrogen Species." Journal of Clinical Investigation 105(8): 1095-1108. http://www.jci.org/articles/view/8574.

Remmerie, Anneleen, and Charlotte L. Scott. 2018. "Macrophages and Lipid Metabolism." Cellular Immunology 330: 27-42. https://linkinghub.elsevier.com/retrieve/pii/S0008874918300327.

Ruan, Wenjing et al. 2017. "Serum Levels of IGFBP7 Are Elevated during Acute Exacerbation in COPD Patients." International Journal of Chronic Obstructive Pulmonary Disease Volume 12: 1775-80. https://www.dovepress.com/serum-levels-of-igfbp7-are-elevated-during-acute-exacerbationin-copd--peer-reviewed-article-COPD.

Shattuck-Heidorn, Heather et al. 2016. "Energetics and the Immune System: Trade-Offs Associated with Non-Acute Levels of CRP in Adolescent Gambian Girls." Evolution, Medicine, and Public Health: eow034. https://academic.oup.com/emph/article-lookup/doi/10.1093/emph/eow034.

Soeters, Maarten R., and Peter B. Soeters. 2012. "The Evolutionary Benefit of Insulin Resistance." Clinical Nutrition 31(6): 1002-7. https://linkinghub.elsevier.com/retrieve/pii/S0261561412001112.

Sparks, Janet D, and Henry H Dong. 2009. "FoxO1 and Hepatic Lipid Metabolism." Current Opinion in Lipidology 20(3): 217-26. http://journals.Iww.com/00041433-200906000-00012.

Straub, Rainer H. 2014. "Insulin Resistance, Selfish Brain, and Selfish Immune System: An Evolutionarily Positively Selected Program Used in Chronic Inflammatory Diseases." Arthritis Research \& Therapy 16(Suppl 2): S4. http://arthritisresearch.biomedcentral.com/articles/10.1186/ar4688.

Teng, Ooiean, Candice Ke En Ang, and Xue Li Guan. 2017. "Macrophage-Bacteria Interactions-A Lipid-Centric Relationship." Frontiers in Immunology 8. http://journal.frontiersin.org/article/10.3389/fimmu.2017.01836/full.

Warburg, Otto. 1925. "Über Den Stoffwechsel Der Carcinomzelle." Klinische Wochenschrift 4(12): 534-36. http://link.springer.com/10.1007/BF01726151.

Zmora, Niv, Stavros Bashiardes, Maayan Levy, and Eran Elinav. 2017. "The Role of the Immune System in Metabolic Health and Disease." Cell Metabolism 25(3): 506-21. https://linkinghub.elsevier.com/retrieve/pii/S1550413117300967. 
Figure 1. Streptococcal infection increases ImpL2 expression in a Hif1 $\alpha$-dependent manner in 812 activated macrophages

813 (A) Expression of ImpL2 mRNA at whole-body level in flies (Crq>GFP) infected with S. pneumoniae 814 (Sp. Infection) and control flies (Buff. Injection) at 0, 3, 6, 9, 12, 15, 18 and 21 hpi. (B) Representative confocal microscopy images of control (left) and infected (right) ImpL2Gal4>UASmCherry individuals imaged at $24 \mathrm{hpi}$, from Z stack of 7 layers, autofluorescence in the green channel was used to visualize the fly's body. (C) Confocal microscopy images of ImpL2Gal4>UASmCherry hemocyte depicting its phagocytic ability visualized by $S$. aureus-pHrodo-green. The scale bar represents $10 \mu \mathrm{m}$. (D) Confocal microscopy image of ImpL2Gal4>UASmCherry hemocyte actively interacting with GFPlabeled S. pneumoniae (Sp-GFP) ex vivo. The scale bar represents $13 \mu \mathrm{m}$. (E) Number of ImpL2positive macrophages in the thorax of control and infected adult flies (ImpL2Gal4>UASmCherry) at 24hpi. (F) Expression of ImpL2 mRNA (using primers targeting all transcript variants) in hemocytes isolated from control and infected Crq>GFP flies at 24hpi. (G) Gene expression of ImpL2-RA (left), ImpL2-RB and ImpL2-RD (middle) and ImpL2-RC (right) transcript variants in either hemocytes, fat body or thorax muscles dissected from control and infected $\mathrm{Crq}>\mathrm{GFP}$ flies at $24 \mathrm{hpi}$. (H) Gene expression of ImpL2 (using primers targeting all transcript variants) and ImpL2-RA in hemocytes isolated from control and infected Crq>GFP flies with and without hemocyte-specific Hif1 $\alpha$ knockdown at $24 \mathrm{hpi}$. (I) (I) In silico analysis of hypoxic enhancer activity by $500 \mathrm{bp}$ bins at the $\mathrm{mpL} 2$ locus and visualization of ImpL2 transcript variants (Flybase.org). Each black dot plots a log 2 fold change ( $y$-axis) of the difference in the randomer tag counts mapped to the 500-bp bin between normoxic and hypoxic conditions. The triangles show the position and negative log10 $p$-value ( $y$-axis) of multiple hypoxic (Hif1 $\alpha$ ), immune (Rel) and stress (HSF) response transcription factor binding sites. The close up shows distribution and clustering of individual response elements for the most significant bin. In (A, F, H), expression levels normalized against rp49 are reported as fold change relative to levels of $\mathrm{ImpL2}$ (using primers targeting all transcript variants) in PBS-injected Crq $>$ GFP controls, which were arbitrarily set to 1 . In (G), expression levels normalized against rp49 are reported as fold change relative to levels of ImpL2-RA in hemocytes of PBS-injected Crq>GFP controls for all three graphs. The individual dots represent biological replicates. Values are mean $\pm S D$, asterisks mark statistically significant differences ( $\left.{ }^{*} p<0.05 ;{ }^{* *} p<0.01 ;{ }^{* * *} p<0.001\right)$. Hif $1 \alpha$, hypoxiainducible factor $1 \alpha$; Rel, relish; HSF, heat shock factor.

Figure 1-figure supplement 1. Efficiency of genetic manipulations of Hif1 $\alpha$ and ImpL2 in Drosophila macrophages

(A) Gene expression of Hif $1 \alpha$ in hemocytes of Buff-injected flies and S. pneumoniae-infected flies with or without macrophage-specific Hif1 $\alpha$ knockdown. (B) ImpL2 gene expression measured at the whole organism level in flies with macrophage-specific knockdown and overexpression of ImpL2 and their respective controls, either with ImpL2 constructs only or with Gal4 drivers only. Expression levels normalized against $\mathrm{rp} 49$ are presented as a fold change relative to levels of $I \mathrm{mpL} 2$ in $w$; ImpL2 $2^{\text {RNAi }}$ control and Hif1 $\alpha$ in uninfected Hml>Gal4, UAS-GFP $x$ TRiP ${ }^{\text {control }}$, respectively, which were arbitrarily set to 1 . Individual dots represent biological replicates. Values are mean $\pm S D$, asterisks mark statistically significant differences ( $\left.{ }^{*} p<0.05 ;{ }^{* *} p<0.01 ;{ }^{* *} p<0.001\right)$. 
853 Figure 1-figure supplement 2. ImpL2-RA regulatory sequence with transcription factor binding sites

854 500-bp long sequence upstream of ImpL2-RA transcriptional start site (as shown on map) with

855 marked binding sites for Hif1 $\alpha$, Rel and HSF1. Hif1 $\alpha$, hypoxia-inducible factor $1 \alpha$; Rel, relish; HSF1,

856 heat shock factor 1 .

857

Figure 2. Macrophage-derived ImpL2 regulates changes in adipose tissue metabolism induced by infection

(A) Triglyceride concentrations in PBS-injected and S.p.-infected flies with macrophage-specific $1 \mathrm{mpL} 2$ knockdown $\left(I \mathrm{mpL} 2^{\mathrm{RNAi}}\right)$, overexpression $\left(\mathrm{ImpL} 2^{\mathrm{CDS}}\right)$ and their respective controls (TRiP ${ }^{\text {control }}, \mathrm{w}^{1118}$ ) at whole-body level at $24 \mathrm{hpi}$. (B-D) Average size (B) and number (C) of lipid droplets and percentage of area occupied (D) in the fat body of PBS-injected and S.p.-infected flies with macrophage-specific ImpL2 knockdown (ImpL2 ${ }^{\mathrm{RNAi}}$ ), overexpression $\left(\mathrm{ImpL}^{\mathrm{CDS}}\right.$ ) and their respective controls (TRiP ${ }^{\text {control }}$, $\mathrm{w}^{1118}$ ) at $24 \mathrm{hpi}$. (E) Representative confocal microscopy images of dissected fat body of PBS-injected and S.p.-infected flies with macrophage-specific $1 \mathrm{mpL2}$ knockdown $\left(\operatorname{ImpL2}{ }^{\mathrm{RNAi}}\right)$, overexpression $\left(I \mathrm{mpL2}{ }^{\mathrm{CDS}}\right.$ ) and their respective controls (TRiP ${ }^{\text {control }}, \mathrm{w}^{1118}$ ) at $24 \mathrm{hpi}$, stained with OilRedO (red), DAPI (cyan) and phalloidin (white). The scale bar represents $20 \mu \mathrm{m}$. (F) Relative proportions of different lipid species in the fat body of PBS-injected and S.p.-infected flies with macrophage-specific ImpL2 knockdown $\left(I \mathrm{mpL} 2^{\mathrm{RNAi}}\right)$, overexpression $\left(I \mathrm{mpL} \mathrm{C}^{\mathrm{CDS}}\right.$ ) and their respective controls (TRiP ${ }^{\text {control }}, \mathrm{w}^{1118}$ ) at 24 hpi. (G) Glycogen concentration in PBS-injected and S.p.-infected flies with macrophage-specific ImpL2 knockdown (ImpL2 $\left.{ }^{\mathrm{RNAi}}\right)$, overexpression $\left(I \mathrm{mpL} 2^{\mathrm{CDS}}\right.$ ) and their respective controls (TRiP ${ }^{\text {control, }}$, $\mathrm{w}^{1118}$ ) at whole-body level at $24 \mathrm{hpi}$. Metabolite concentrations were normalized to the amount of proteins in each sample and measured in three biological replicates. Values are mean \pm SD, asterisks mark statistically significant differences $\left({ }^{*} p<0.05 ;{ }^{*} p<0.01 ; * * * p<0.001\right)$. (B-D) were quantified from 8 confocal microscopy images for each genotype and treatment, (F) lipidomic analysis was performed in three biological replicates for each genotype and treatment. $P C$, phosphatidylcholine; $P E$, phosphatidylethanolamine; TG, triglycerides; DG, diglycerides; PG, phosphatidylglycerol; PI, phosphatidylinositol; LysoPC, Lyso-phosphatidylcholine; LysoPE, Lyso-phosphatidylethanolamine.

880

Figure 3. Infection-induced autophagy and changes in gene expression in adipose tissue

882

883

884

885

886

887

888

889

890

891

892

893

894

895

896

897
(A) Representative confocal microscopy images of fat body of control and infected flies with Atg8amCherry autophagy reporter (Atg8a, red; DAPI, cyan). The scale bar represents $20 \mu \mathrm{m}$. (B) Graphical representation of log2-fold change in mRNA expression of genes involved in insulin signaling, lipoprotein and lipid metabolism, glycogenolysis and autophagy in the fat body of PBS-injected and S.p.-infected flies with macrophage-specific $I m p L 2$ knockdown (ImpL2 ${ }^{\text {RNAi }}$ ), overexpression $\left(I m p L 2{ }^{\mathrm{CDS}}\right.$ ) and their respective controls (TRiP ${ }^{\text {control }}, \mathrm{w}^{1118}$ ) at $24 \mathrm{hpi}$. The first column (Infection) shows the log2fold change in mRNA expression in S.p-infected flies compared to Buff.-injected control flies (average change for TRiPcontrol and $w^{1118}$ ). The second to fourth columns represent the log2-fold change in mRNA expression compared to the corresponding control for knockdown (ImpL ${ }^{\text {RNAi }} /$ TRiP $^{\text {control }}$ ) and overexpression ( $\mathrm{ImpL2}{ }^{\mathrm{CDS}} / \mathrm{w}^{1118}$ ) in either uninfected (Buff.) or S.p. infected flies. Foxo, forkhead box O; 4EBP, Thor; EIF-4E1, eukaryotic translation initiation factor 4E1; MTP, microsomal triacylglycerol transfer protein; apoLPP, apolipophorin; apoLTP, apolipoprotein lipid transfer particle; Bmm, brummer; Lip4, lipase 4; Hsl, hormone sensitive lipase; Glutaryl-CoA deh., Glutaryl-CoA dehydrogenase; GlyS, glycogen synthase; GlyP, glycogen phosphorylase; Atg1, autophagy-related gene 1; Atg6, autophagy-related gene 6. 

GlyP, Atg1, and Atg6 genes in adipose tissue of flies injected with PBS (black bars) and infected with S. pneumoniae (grey bars) with macrophage-specific ImpL2 knockdown, overexpression and their respective controls; all genotypes depicted below the x-axis were crossed with $\mathrm{Hml}>$ Gal4; TubGal80 $0^{T S}$. Expression levels normalized against $\mathrm{rp} 49$ are present as a fold-change relative to the levels in the PBS-injected TRiP ${ }^{\text {control }}$, which was arbitrarily set to 1 . The individual dots represent biological replicates. Values are mean $\pm S D$, asterisks mark statistically significant differences

$906\left({ }^{*} p<0.05 ; *{ }^{*} p<0.01 ; * * *<0.001\right)$.

907

Figure 4. Macrophage-derived ImpL2 affects adipose tissue metabolism via Foxo activity

Representative confocal microscopy images of Foxo immunolocalization in the fat body of PBSinjected and S.p.-infected flies with macrophage-specific $\operatorname{lmpL2}$ knockdown ( $I m p L 2^{\text {RNAi }}$ ), overexpression ( $\left(\mathrm{mpL} 2^{\mathrm{CDS}}\right.$ ) and their respective controls (TRiP ${ }^{\text {control }}, \mathrm{w}^{1118}$ ) at $24 \mathrm{hpi}$; anti-dFoxo antibody, green; DAPI, cyan. Histograms of Foxo cellular localization represent Foxo and DAPI signal intensity in sections indicated by a white freehand line. The scale bar represents $20 \mu \mathrm{m}$.

Figure 5. Macrophage-derived ImpL2 increases circulating carbohydrates and cholesterols to be utilized by activated macrophages

(A-D) Concentrations of circulating glucose (A), trehalose (B), cholesterol (C) and cholesteryl-ester (D) in the hemolymph of PBS-injected and S.p.-infected flies with macrophage-specific ImpL2 knockdown $\left(\mathrm{ImpL}^{\mathrm{RNAi}}\right.$ ), overexpression (ImpL2 ${ }^{\mathrm{CDS}}$ ) and their respective controls (TRiP ${ }^{\mathrm{Control}}, \mathrm{w}^{1118}$ ) at $24 \mathrm{hpi}$. (E-H) Concentrations of glucose $(\mathbf{E})$, trehalose $(\mathbf{F})$, cholesterol $(\mathbf{G})$ and cholesteryl-ester $(\mathbf{H})$ in hemocytes of PBS-injected and S.p.-infected flies with macrophage-specific ImpL2 knockdown (ImpL2 ${ }^{\mathrm{RNAi}}$ ), overexpression (ImpL2 ${ }^{\mathrm{CDS}}$ ), and their respective controls (TRiP ${ }^{\text {control }}, \mathrm{w}^{1118}$ ) at $24 \mathrm{hpi}$. (I) Proportional occurrence of macrophages containing at least one lipid droplet (stained by OilRed 0 ), counted in at least fifty of Crq>GFP positive cells. (J) Representative confocal microscopy image of macrophages ( $\mathrm{Crq}>\mathrm{GFP}$ ) containing lipid droplets; neutral lipids were stained with OilRed $\mathrm{O}$ (red). The scale bar represents $10 \mu \mathrm{m}$. The image represents a Z-stack consisting of a maximum projection of 5 layers. (K) Representative confocal microscopy image depicting the ability of macrophage ( $\mathrm{Crq}>\mathrm{GFP}$ ) to endocytose lipoproteins (human LDL-pHrodo, red). The scale bar represents $13 \mu \mathrm{m}$. (A-H) Metabolite concentrations were normalized to the amount of proteins in each sample. Metabolic measurements were performed in three biological replicates. Values are mean $\pm S D$, asterisks mark statistically significant differences $\left({ }^{*} p<0.05 ;{ }^{* *} p<0.01 ; * * p<0.001\right)$.

Figure 6. Macrophage-derived ImpL2 is important for resistance to bacterial infection

(A, C) Survival rate of S.p.-infected and PBS-injected flies with macrophage-specific ImpL2 knockdown $\left(I m p L 2^{\text {RNAi }}\right.$ (A), overexpression ( $I m p L 2^{\text {CDS }}$ ) (C) and their respective controls (TRiP ${ }^{\text {control }}, w^{1118}$ ). Three independent experiments were performed and combined into one survival curve; the number of individuals per replicate was at least 500 for each genotype. (B, D) Pathogen load in S.p.-infected and PBS-injected flies with knockdown $\left(I \mathrm{mpL} 2^{\mathrm{RNAi}}\right)(\mathrm{B})$, overexpression $\left(I \mathrm{mpL} 2^{\mathrm{CDS}}\right)(\mathrm{D})$ and their respective controls (TRiP ${ }^{\text {control }}, \mathrm{w}^{1118}$ ) at 24 and $48 \mathrm{hpi}$. The individual dots in the plot represent the number of 
940 bacteria (colony forming units-CFUs) in one fly. The data show results combined from three

941 independent biological replicates. (E) Confocal microscopy image depicting the ability of a

942 macrophage ( $\mathrm{Crq}>\mathrm{GFP}$ ) to phagocytose an invading pathogen (S. aureus-pHrodo-red). The scale bar

943 represents $10 \mu \mathrm{m}$. The image represents a Z-stack consisting of a maximum projection of 5 layers. (F)

944 Phagocytic rate calculated for S.p.-infected flies with knockdown (ImpL2 $\left.{ }^{\text {RNAi }}\right)$, overexpression

$945\left(I \mathrm{mpL2}{ }^{\mathrm{CDS}}\right)$, and their respective controls (TRiP ${ }^{\text {control }}, \mathrm{w}^{1118}$ ); each dot in the plot represents the

946 number of phagocytic events per macrophage (Crq>GFP). The results are combined from three

947 biological replicates. Values are mean \pm SD, asterisks mark statistically significant differences

$948 \quad\left({ }^{*} p<0.05 ; * * p<0.01 ; * * p<0.001\right)$.

949

950

951

Figure 7. Maladaptive effects of ImpL2 when overexpressed during development and in chronic infection

(A) Representative images of a developmental defect of the dorsal closure in the abdomen of a fly with macrophage-specific $\mathrm{ImpL} 2$ overexpression throughout development ( $\mathrm{Crq}>\mathrm{Gal} / 4>\operatorname{ImpL2}{ }^{\mathrm{CDS}}$ ) (left), and melanized spots in a fly with temporal (48 hours), macrophage-specific $1 \mathrm{mpL} 2$ overexpression $\left(\mathrm{Hml}>\mathrm{Gal} 4\right.$; $\mathrm{Gal} 80^{\mathrm{TS}}>\mathrm{ImpL} 2^{\mathrm{CDS}}$ ) (middle), with the aortic region (upper right with arrows pointing to melanized spots) and melanized spot (lower right) at higher magnification. (B, D) Survival rate of $L$. monocytogenes-infected and PBS-injected flies with macrophage-specific ImpL2 knockdown $\left(I m p L 2^{\text {RNAi }}\right)(B)$, overexpression (ImpL2 $\left.{ }^{\mathrm{CDS}}\right)$ (D) and their respective controls (TRiP ${ }^{\text {control }}, w^{1118}$ ). Three independent experiments were performed and combined into each survival curve; the number of individuals per replicate was at least 500 for each genotype. (C, E) Pathogen load of $L$. monocytogenes in flies with macrophage-specific ImpL2 knockdown $\left(I m p L 2^{\text {RNAi }}\right.$ (C), overexpression $\left(\mathrm{ImpL}^{\mathrm{CDS}}\right)(\mathrm{E})$ and their respective controls (TRiP ${ }^{\text {control }}, \mathrm{w}^{1118}$ ) shownas either total load or only intracellular Listeria subpopulation at $24 \mathrm{hpi}$ and at 12 days post-infection. The individual dots in the plot represent the number of bacteria (colony forming units-CFUs) in one fly. The data show results combined from three independent biological replicates. Values are mean $\pm S D$, asterisks mark statistically significant differences $\left({ }^{*} p<0.05 ;{ }^{* *} p<0.01 ;{ }^{* * *} p<0.001\right)$.

Figure 8. IGFBP7, the mammalian homolog of $1 \mathrm{mpL2}$, is produced in human macrophages in response to $S$. pneumoniae in vitro

(A) Confocal microscopy images of PMA-activated control THP-1 cells (left) and PMA-activated THP-1 cells actively interacting with GFP-labeled S. pneumoniae (green) in vitro (right). The scale bar represents $10 \mu \mathrm{m}$. The image on the right is a maximum projection of 3 layers. (B) Gene expression of IGFBP7, IGFBP3 and IGFBP4 in PMA-activated THP-1 cells 24 hours after exposure to $S$. pne umoniae in vitro. Expression levels normalized against ACTB are shown as a fold change relative to levels of IGFBP7 in controls that were arbitrarily set to 1 . Values are mean \pm SD, asterisks mark statistically significant differences $\left({ }^{*} p<0.05 ;{ }^{* *} p<0.01 ;{ }^{* *} p<0.001\right)$.

977

\section{Figure 9. Schematic representation of the proposed model of ImpL2 function during infection}

979 Upon recognition of the bacteria, the macrophages switch to Hif1 $\alpha$-driven aerobic glycolysis. Hif1 $\alpha$

980 also induces the expression of the ImpL2-RA isoform by binding to hypoxia response elements in the promotor of the ImpL2 gene. Macrophage-derived ImpL2 then remotely activates Foxo re-

982 localization to the nucleus in the fat body, leading to the expression of metabolic Foxo-target genes. 
bioRxiv preprint doi: https://doi.org/10.1101/2020.09.24.311670; this version posted September 25, 2020. The copyright holder for this preprint (which was not certified by peer review) is the author/funder, who has granted bioRxiv a license to display the preprint in perpetuity. It is made available under aCC-BY-NC-ND 4.0 International license.

983 Mobilized sources, manifested by hyperlipidemia and hyperglycemia in the hemolymph, are

984 subsequently used by activated macrophages. These transient changes in ImpL2-regulated

985 metabolism are essential for efficient bacterial killing and resistance to infection. However, if

986 prolonged, for example during chronic infection, macrophage-derived $1 \mathrm{mpL} 2$ can harm the host by

987 potential wasting. Hif1 $\alpha$, hypoxia-inducible factor $1 \alpha$; HREs, hypoxia response elements; ImpL2,

988 imaginal morphogenesis protein-late 2; Foxo, forkhead box $\mathrm{O}$. 
bioRxiv preprint doi: https://doi.org/10.1101/2020.09.24.311670 this version nosted_Sentember 25 2020 The convriaht holder for this nrenrint

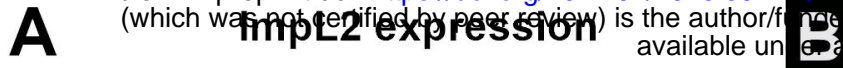
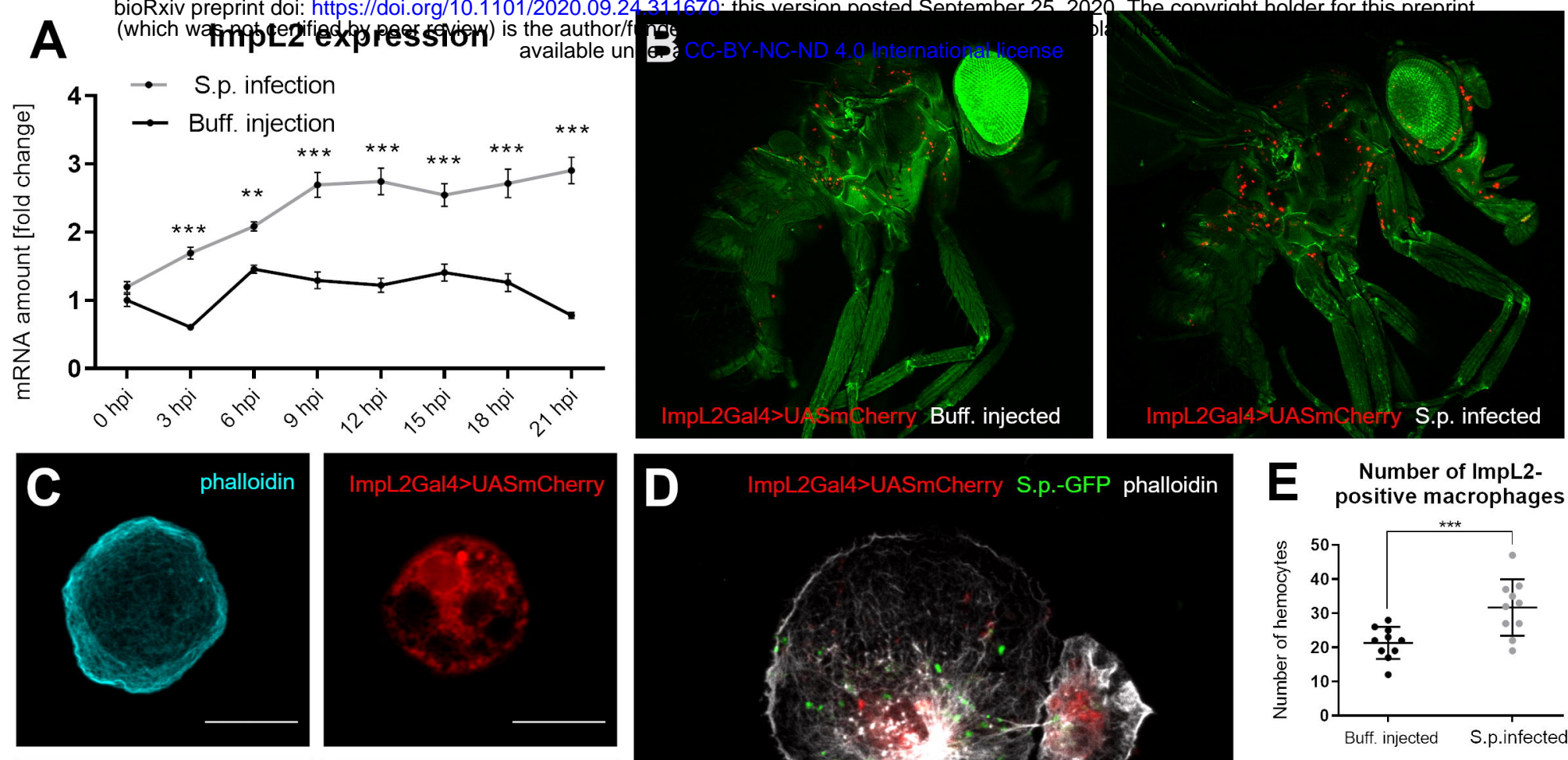

E $\quad \begin{gathered}\text { Number of ImpL2- } \\ \text { positive macrophages }\end{gathered}$
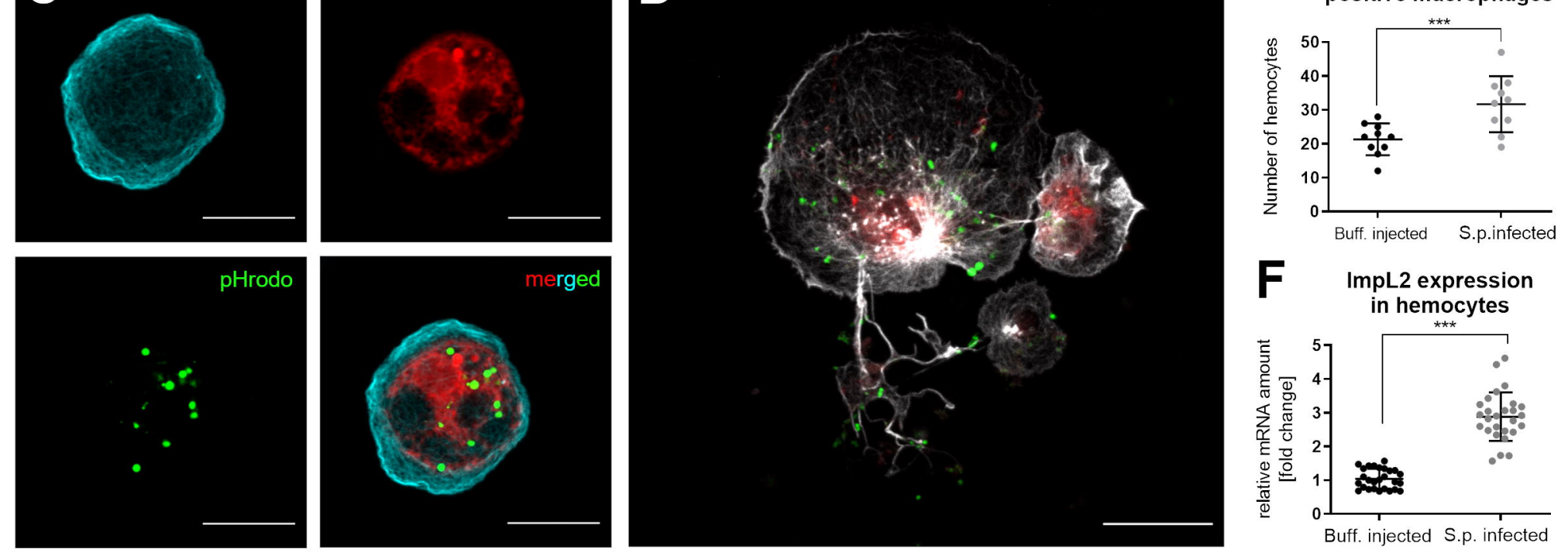

G
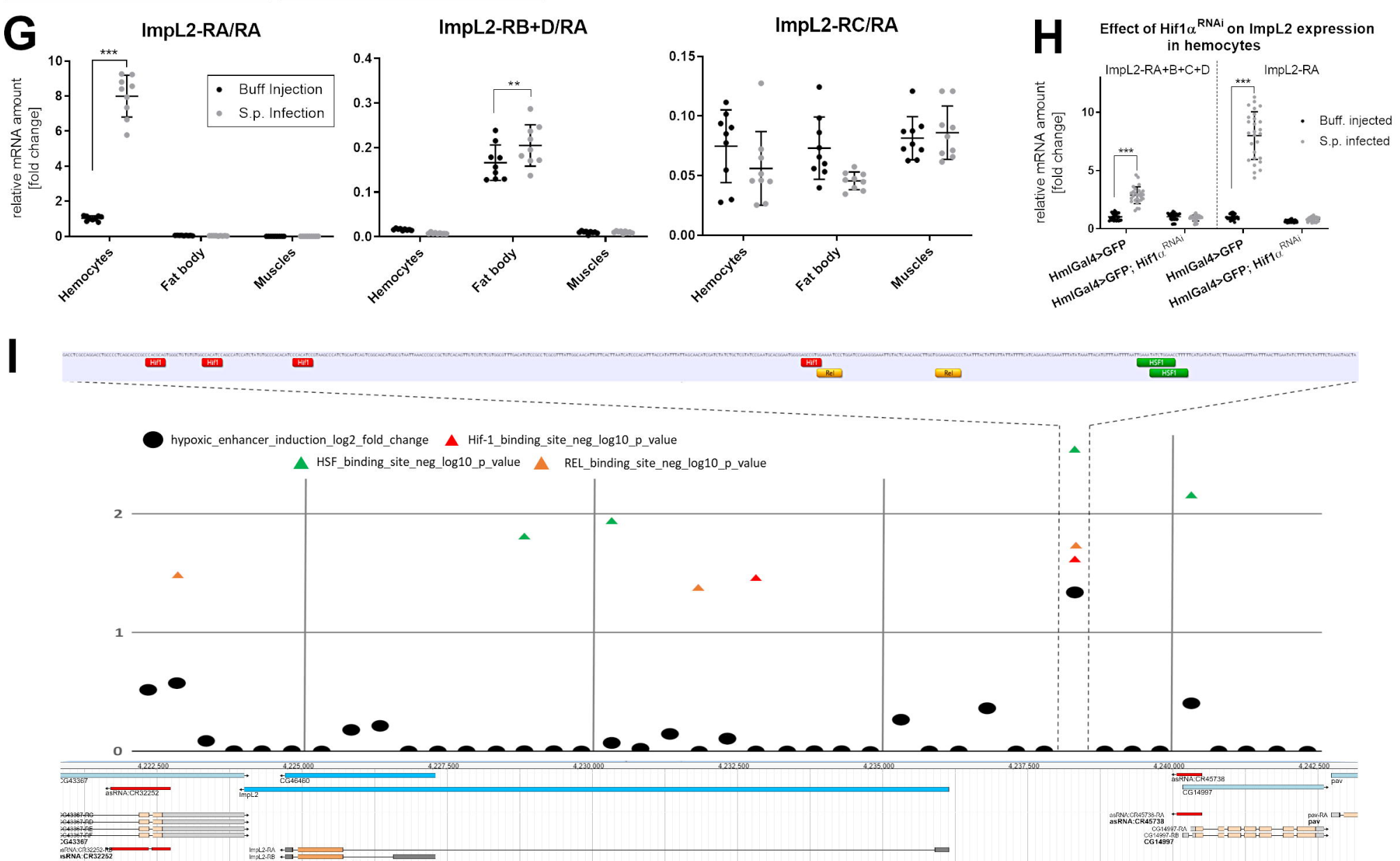

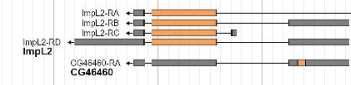



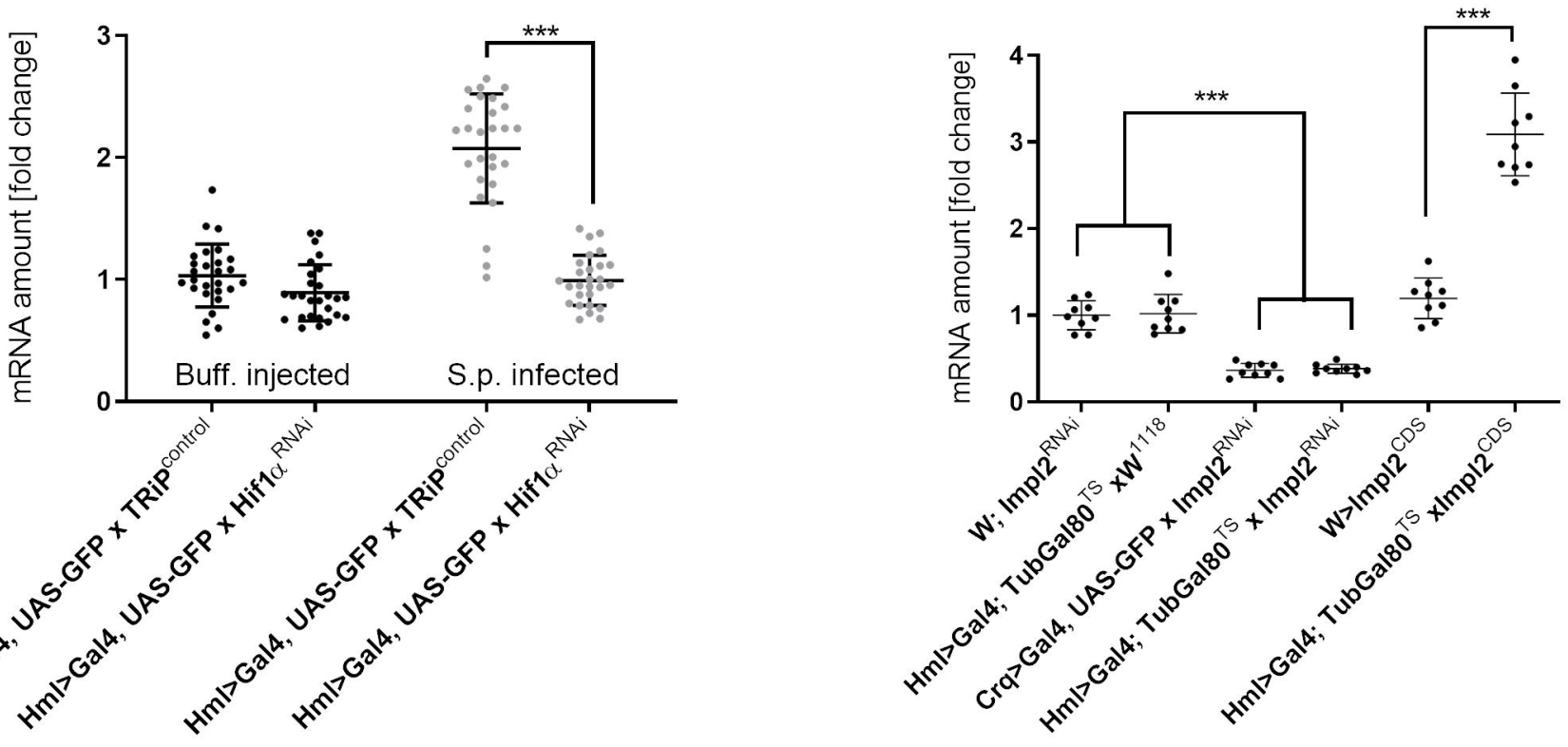
bioRxiv preprint doi: https://doi.org/10.1101/2020.09.24.311670; this version posted September 25, 2020. The copyright holder for this preprint (which was not certified by peer review) is the author/funder, who has granted bioRxiv a license to display the preprint in perpetuity. It is made

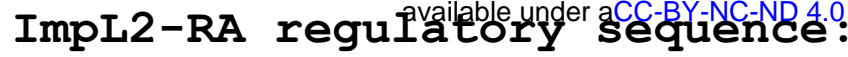

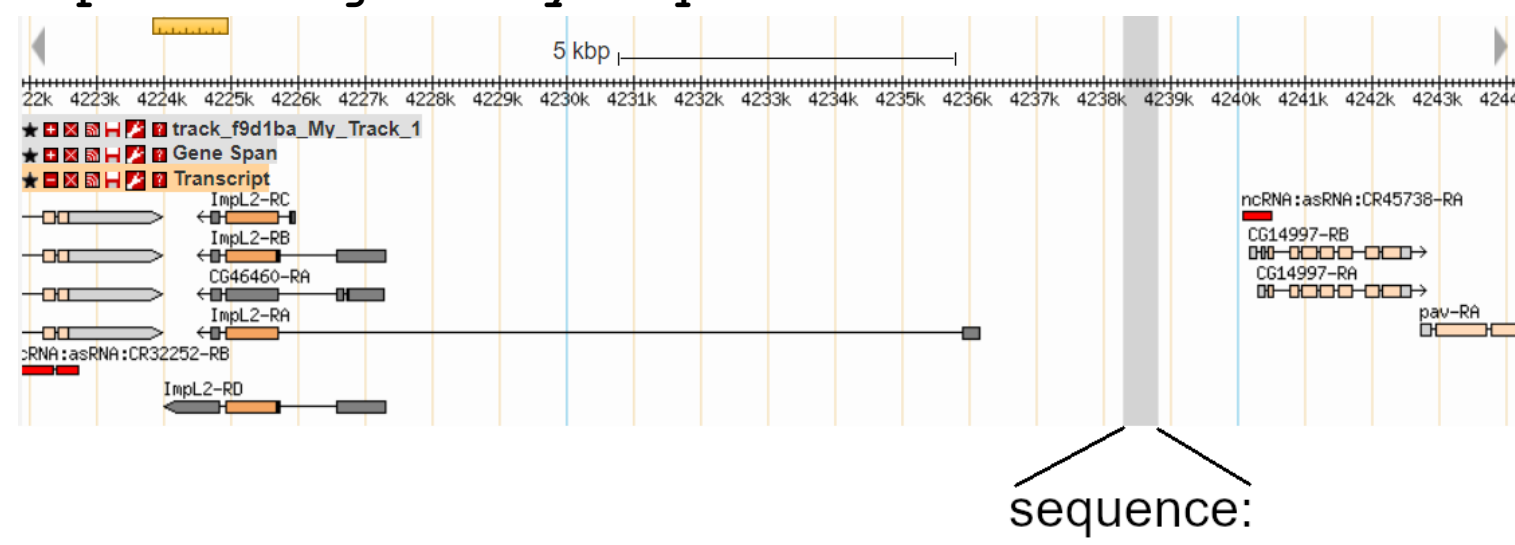

GACCTCGCCAGGACCTGCCCCTCAGCACCCGCCCACGCAGTGGGCTGTGTG

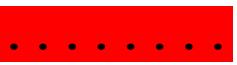

TGGCCACATCCAGCCATCCATCTATGTGCCCACACATCCCACATCCGTAAG
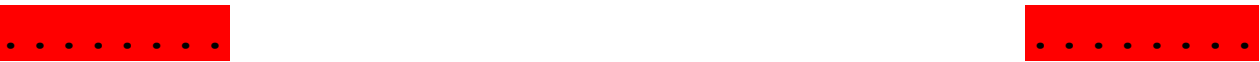

CCCATCTGCAATCAGTCGGCAGCATGGCGTAATTAAACCCGCCGCTGTCAC

AGTTGTCGTCTCGTGGCGTTTGACATGTCCGCCTCGCGTTTATTGGCAACA

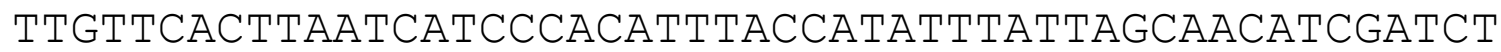

ATCTGCTCGTATCCGAATGCACGGAATGGGGAGCCGTGGAAAATCCCTGGA
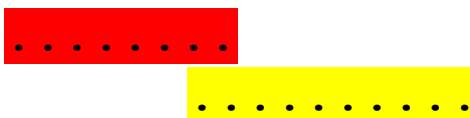

TCCGAAGGGAAATTGTACTCAACAAGCTTGGTGGAAAGACCCCTAATTTAC

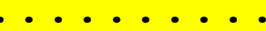

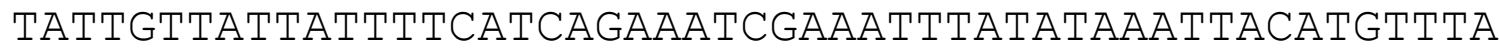

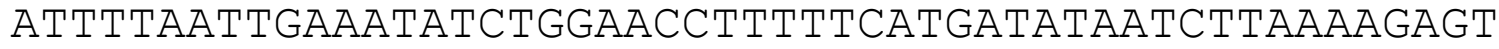

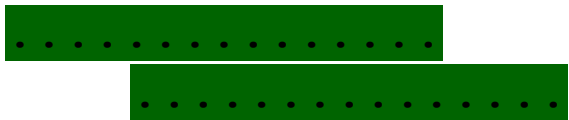

TTAATTAAстTGAAATCTTTATCTATTTCTGAAgTAGCTA

Hifl- $\alpha$ binding site Rel binding site

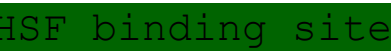




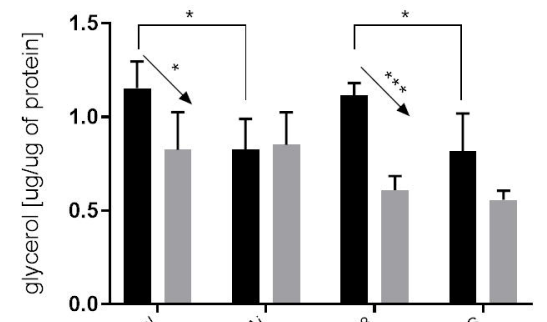

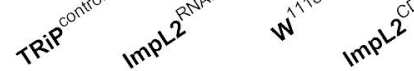

B

Average size of lipid droplets

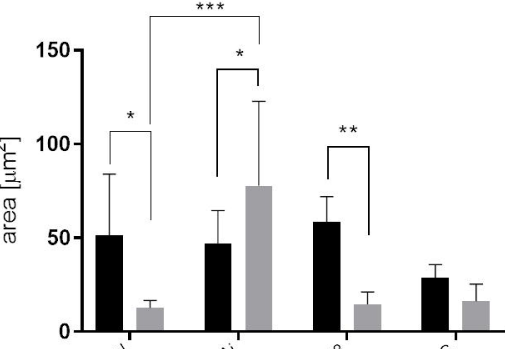

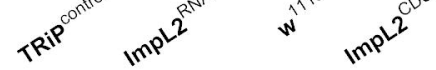

C

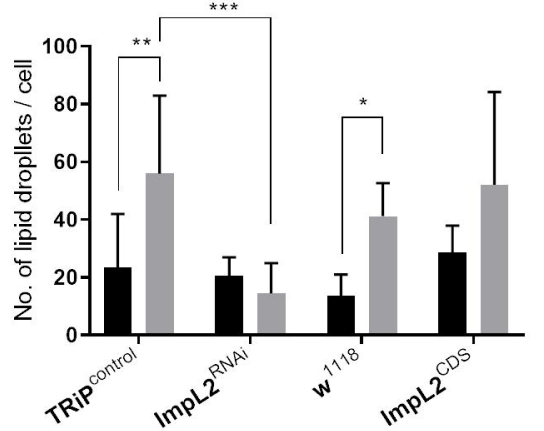

D

Lipid droplet content in adipose tissue

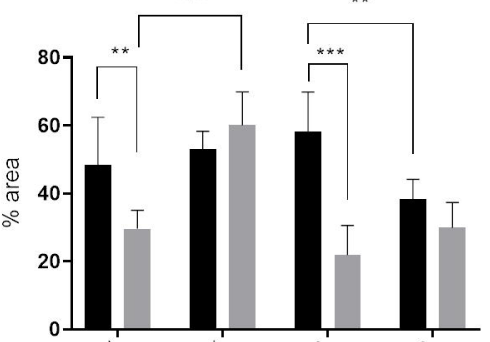

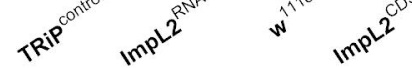

\section{G

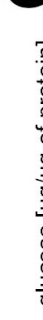

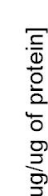

Glycogen whole flies

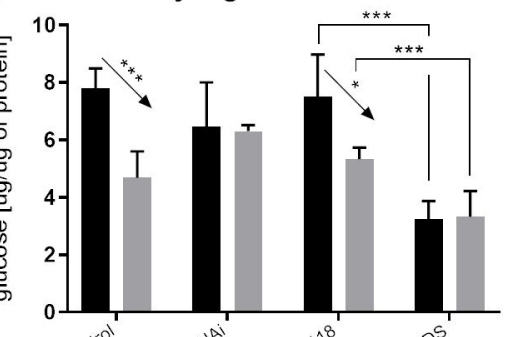

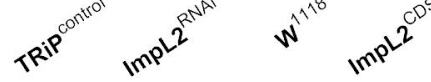

$H m l>G a l 4 ;$ TubGal80 $0^{T S}$

Buff. injected

S.p. infected
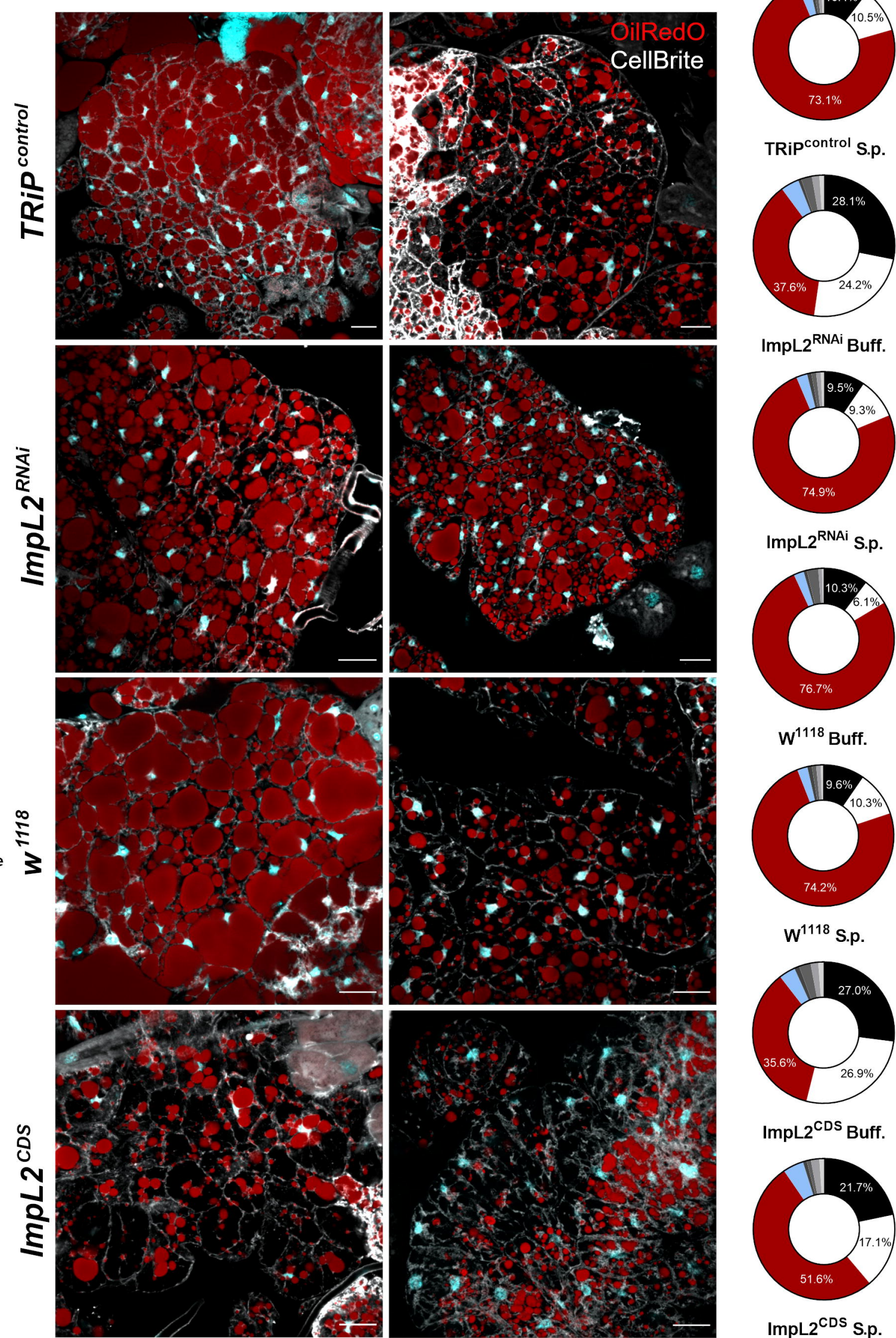

Buff. injected

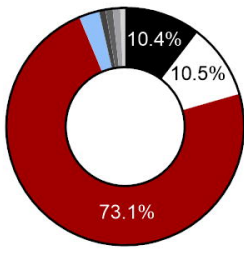

TRiP ${ }^{\text {control }}$ S.p.

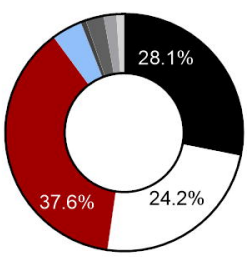

ImpL2 ${ }^{\text {RNAi }}$ Buff.

ImpL2 ${ }^{\text {RNAi }}$ S.p.

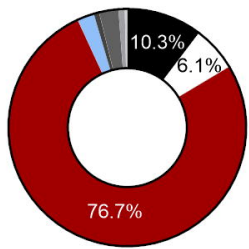

$W^{1118}$ Buff.

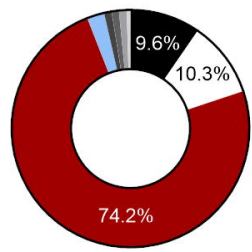

$W^{1118}$ S.p.

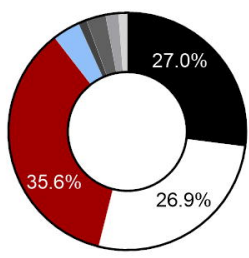

ImpL2 ${ }^{\mathrm{CDS}}$ Buff.

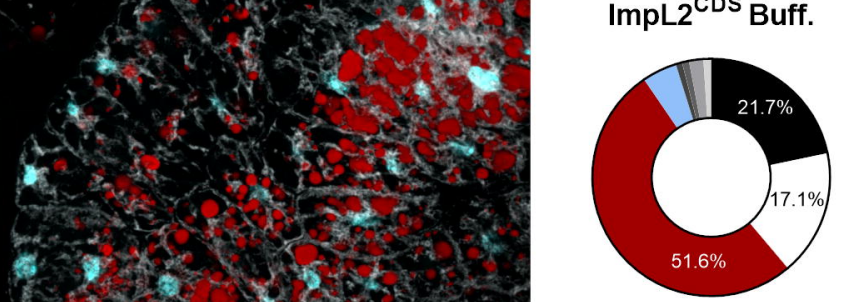

ImpL2 ${ }^{\mathrm{CDS}}$ S.p.
S.p. infected

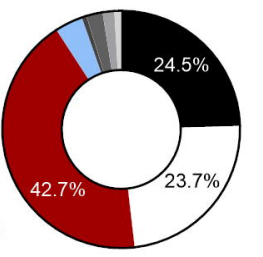




\section{A Atg8a-mCherry DAPI}
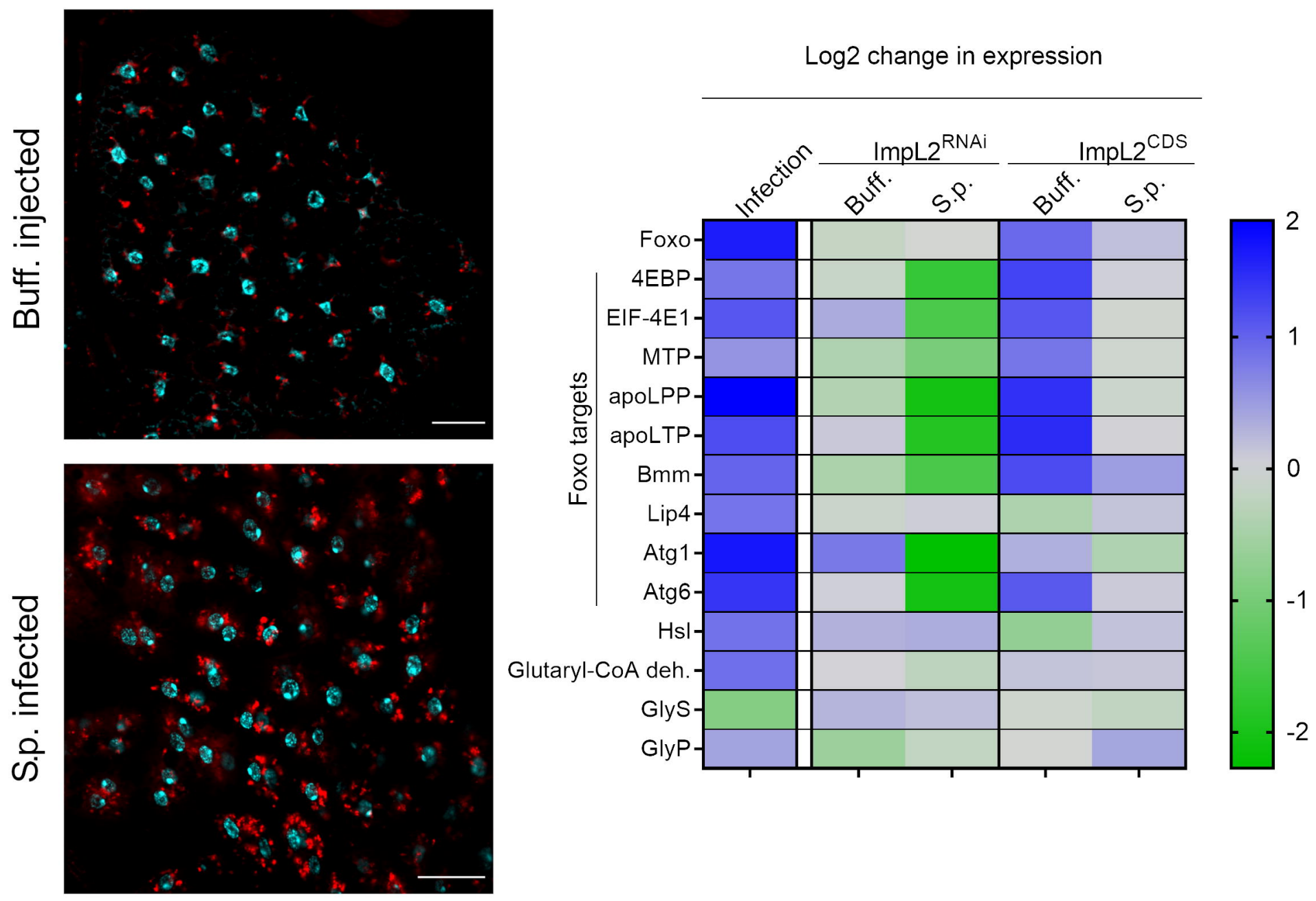


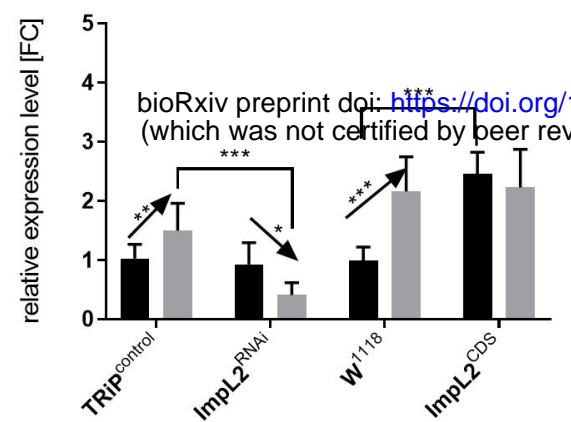

$\bar{v}^{3}$

$\star \star \star \star *$

ㄴ.

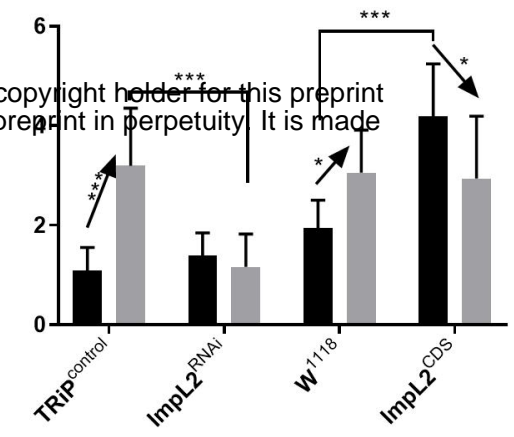

Fat body Glutaryl-CoA deh.

Fat body Lip4

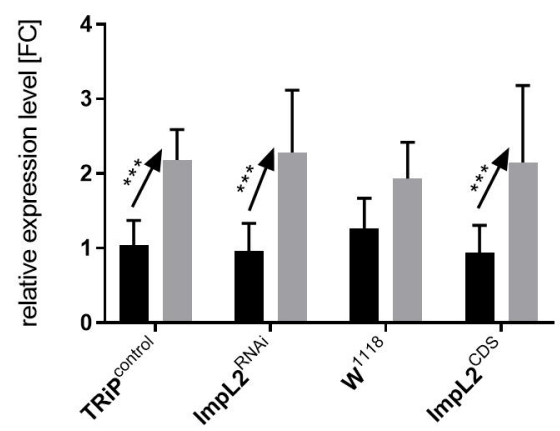

Fat body GlyS

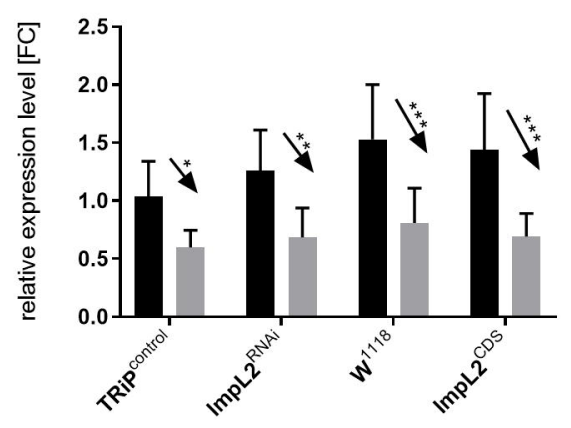

Fat body apoLPP

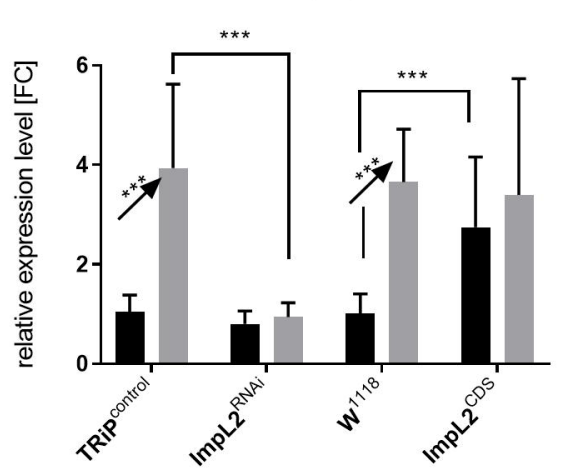

Fat body Atg6

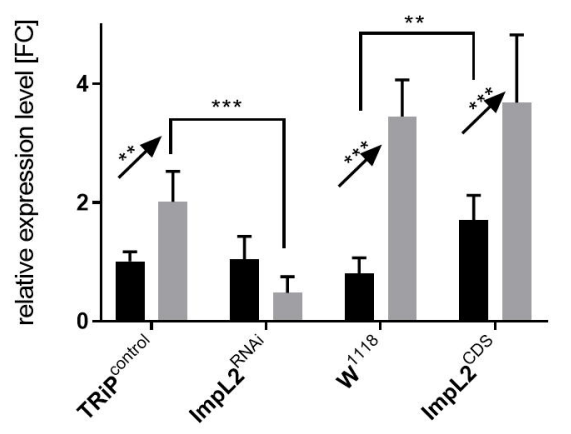

Fat body HSL

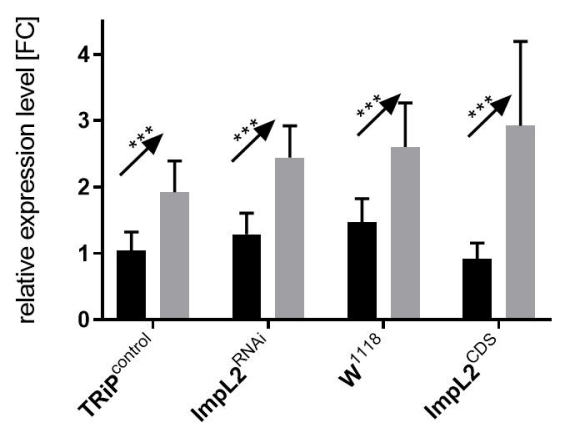

Fat body GlyP

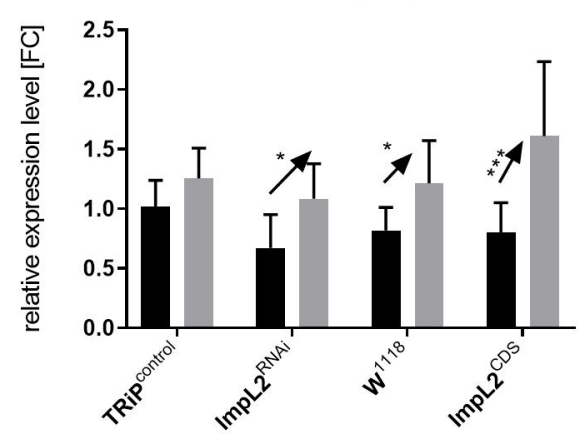

Fat body MTP

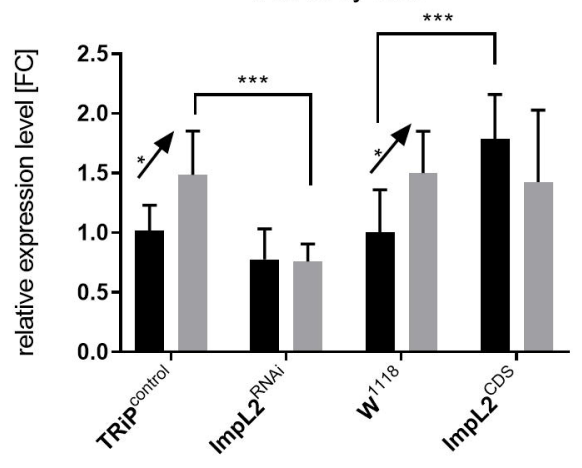

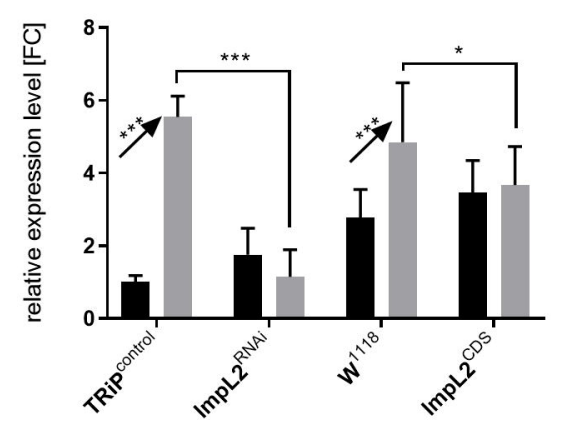




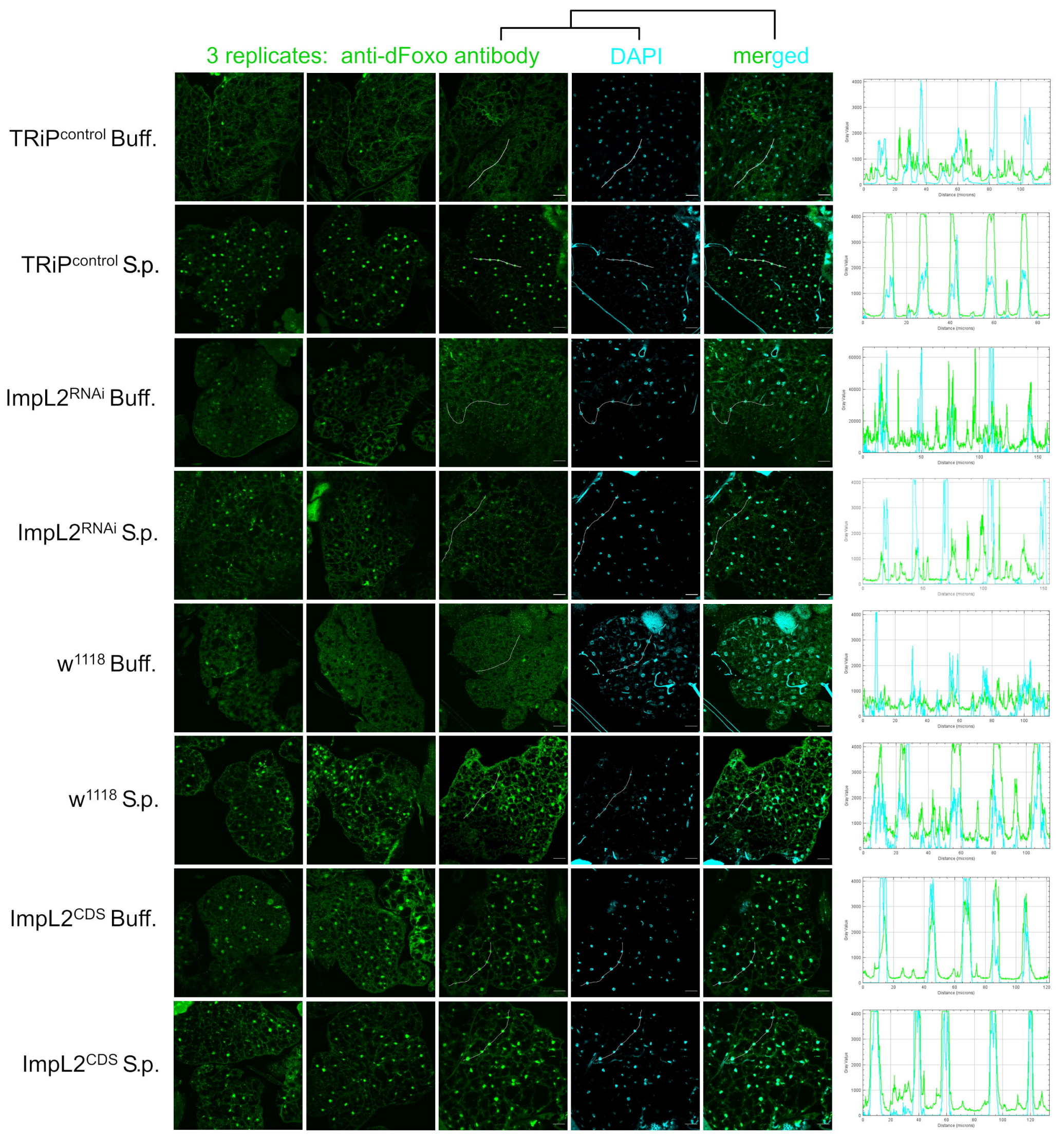


A Glucose hemolymph

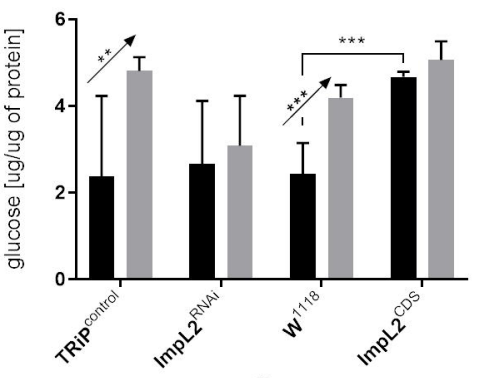

Hml>Gal4; TubGal80 ${ }^{\text {TS }}$

E

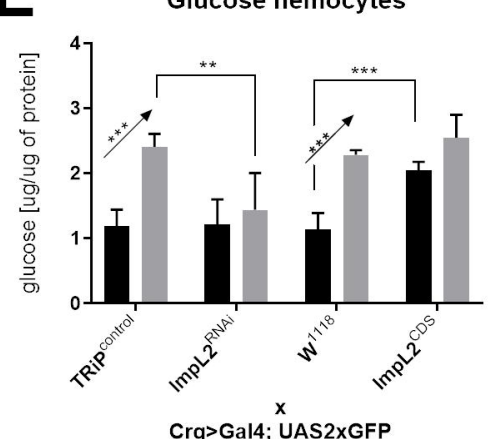

B Trehalose hemolymph C

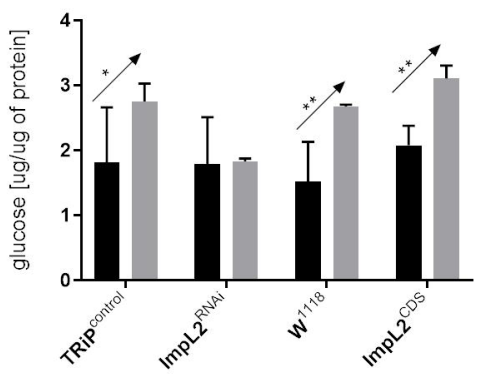

$\mathrm{Hml}>\mathrm{Gal} 4$; TubGal80 ${ }^{\mathrm{TS}}$

F

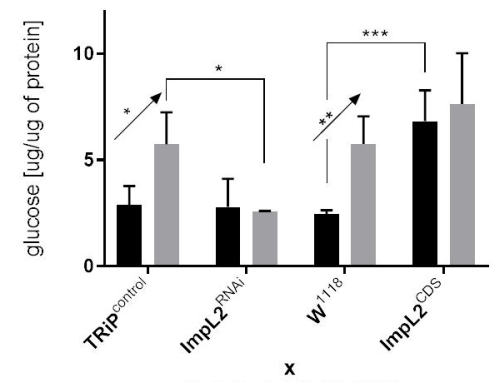

Crq>Gal4; UAS2xGFP

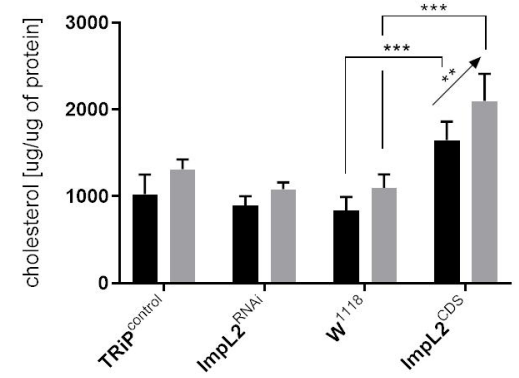

$\mathrm{Hml}>$ Gal4; TubGal80 ${ }^{\mathrm{TS}}$

G

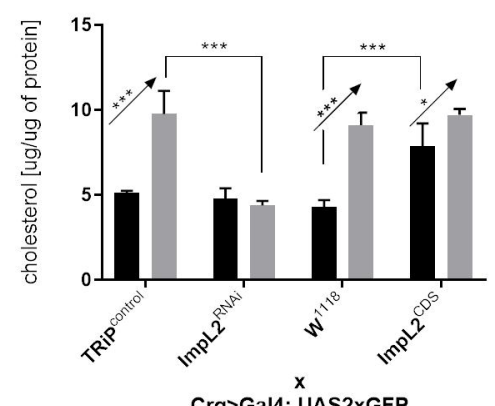

D Cholesteryl-ester hemolymph

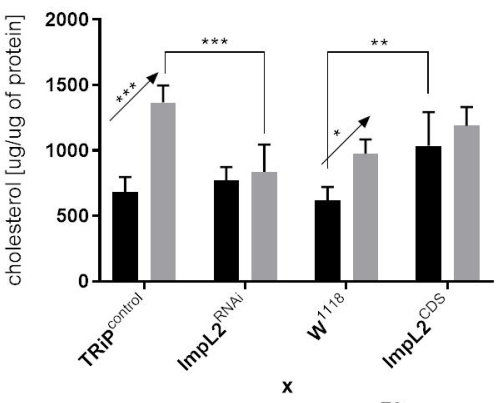

Hml>Gal4; TubGal80 TS

H

Cholesteryl-ester hemocytes

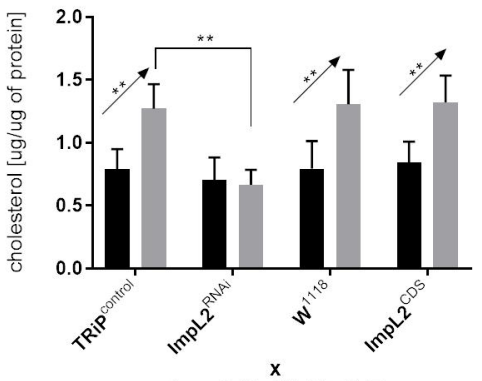

Crq>Gal4; UAS2xGFP
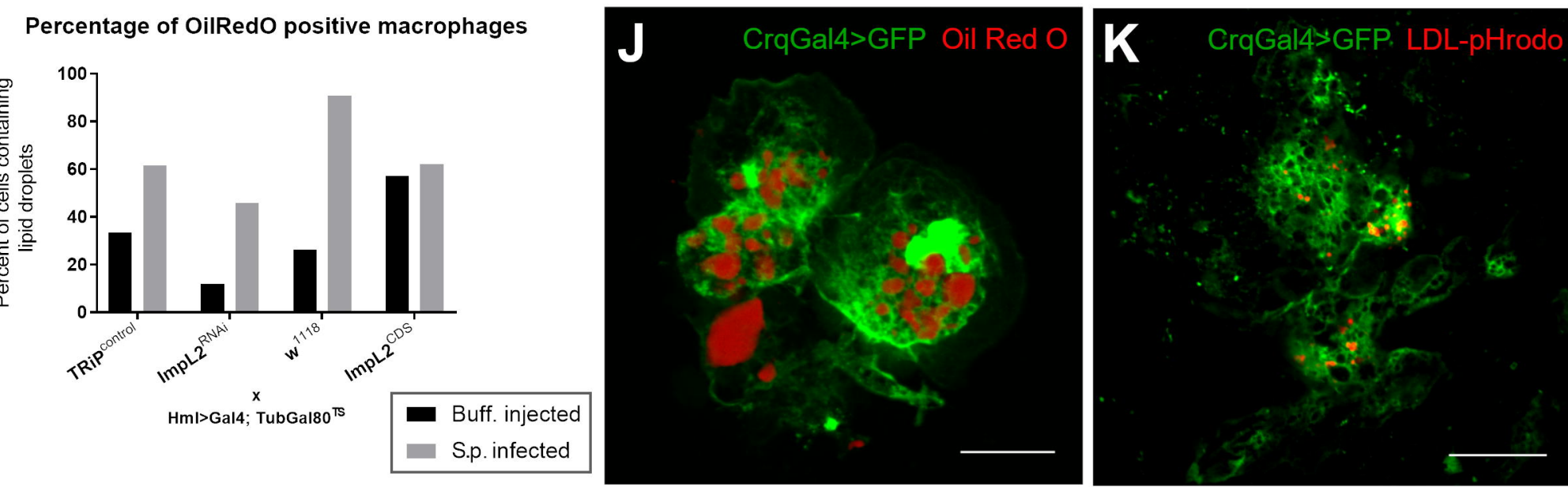


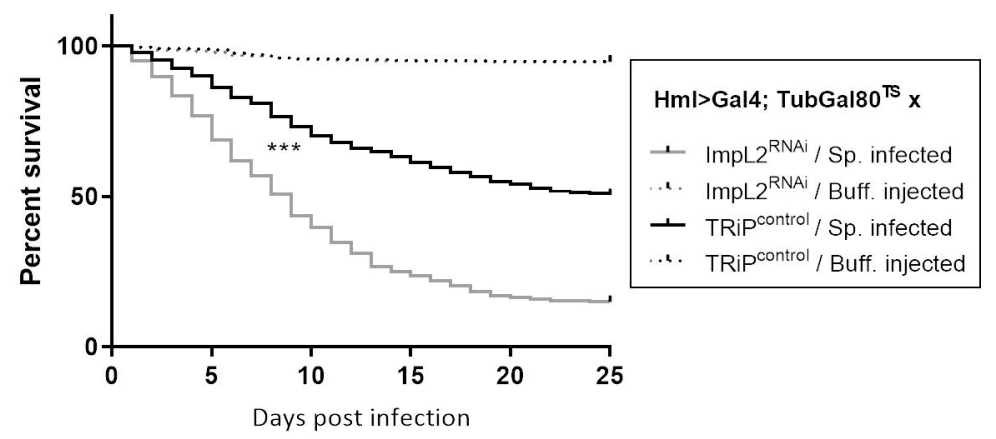

C

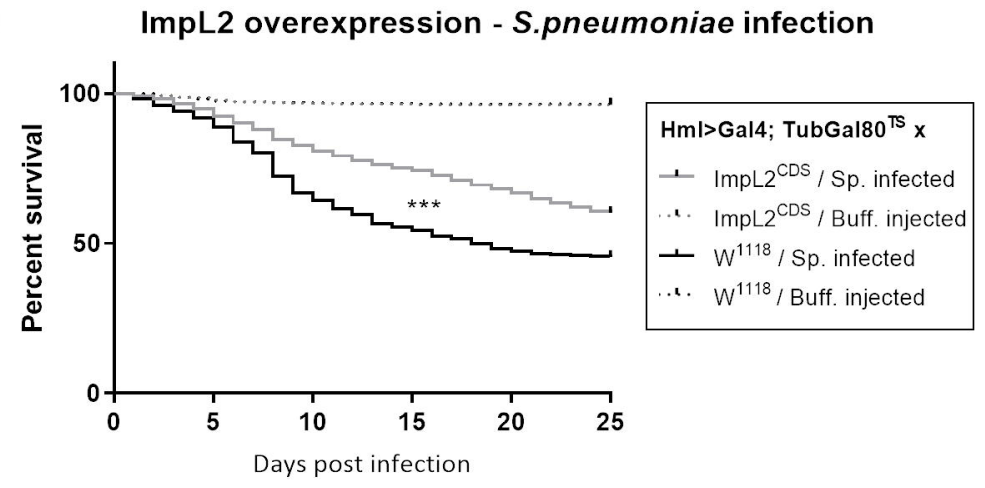

E

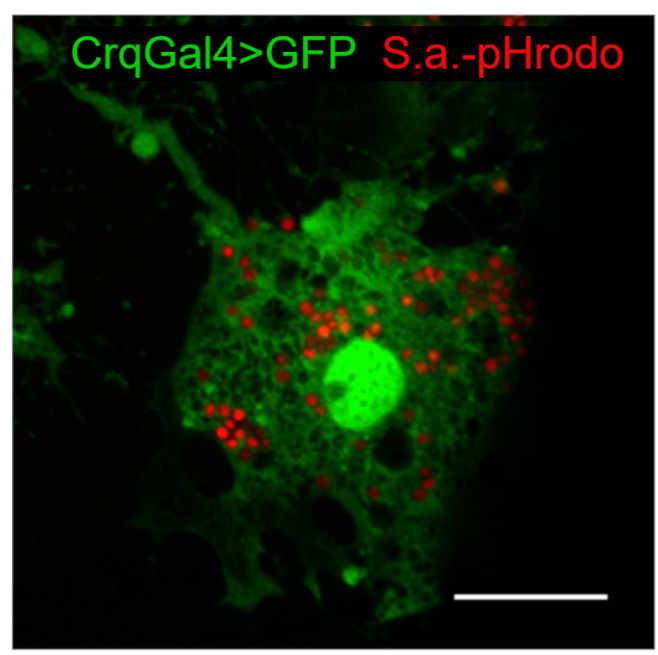

B Pathogen load of S. pneumoniae

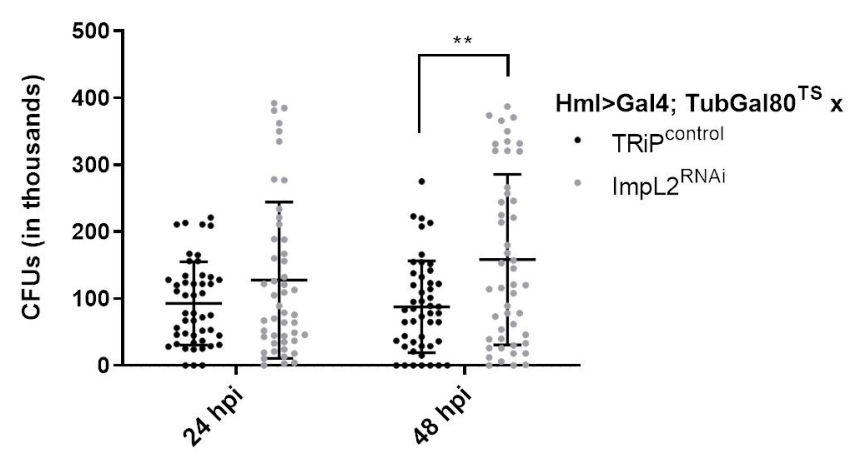

D Pathogen load of S. pneumoniae

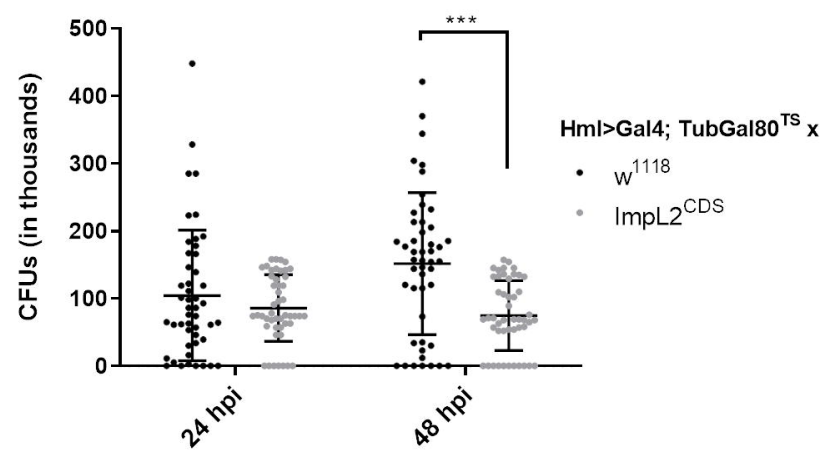

$\mathbf{F}$

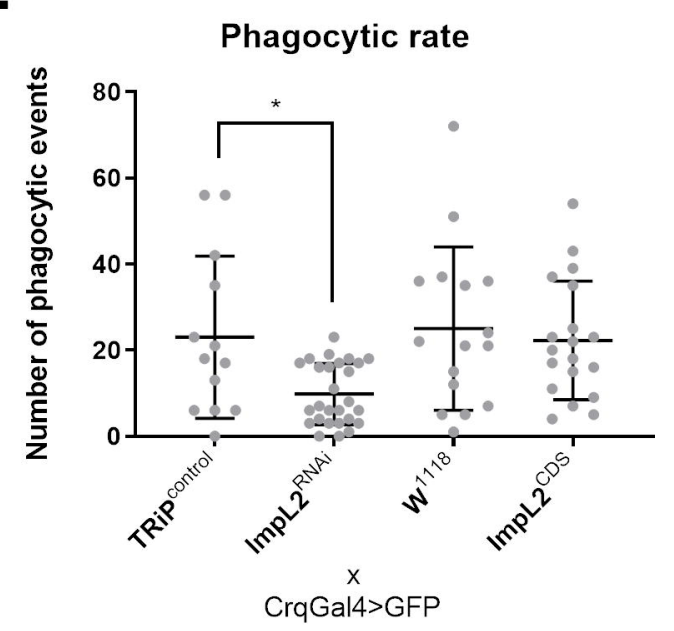




\section{Crq $>$ Gal4}
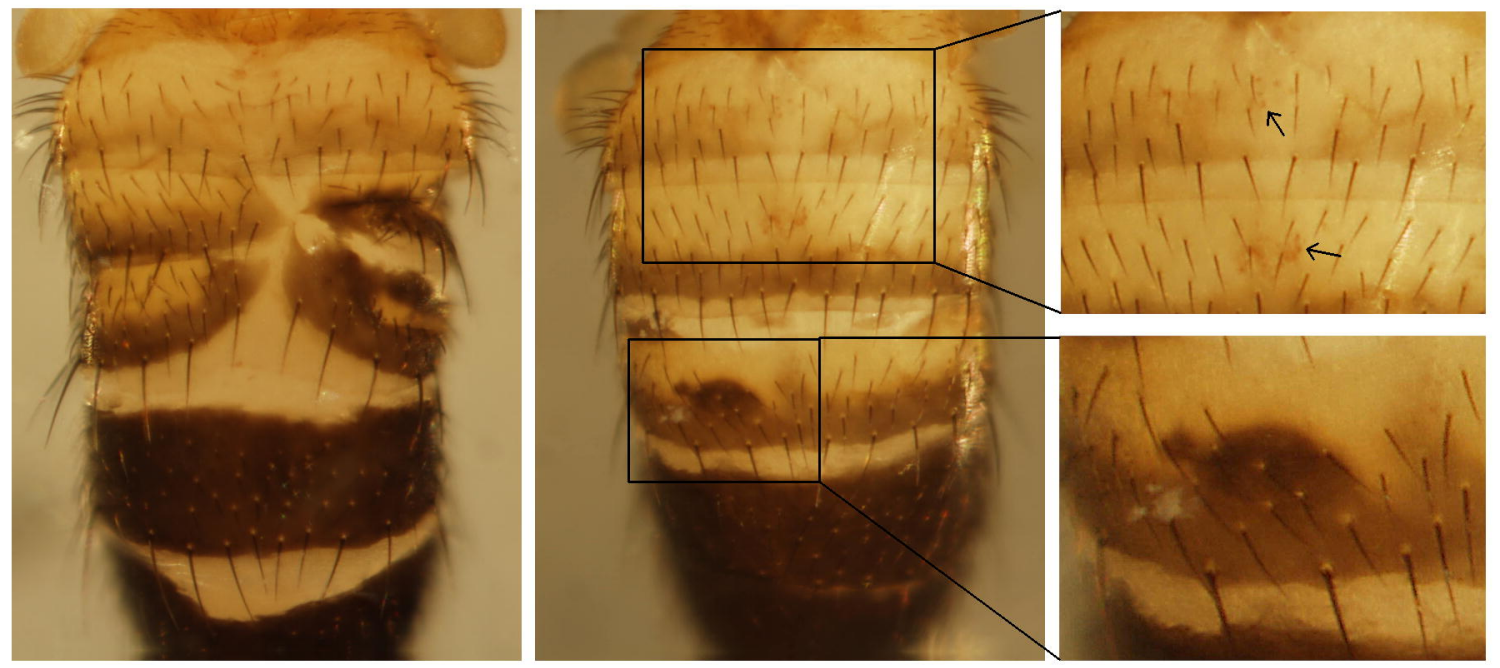

B

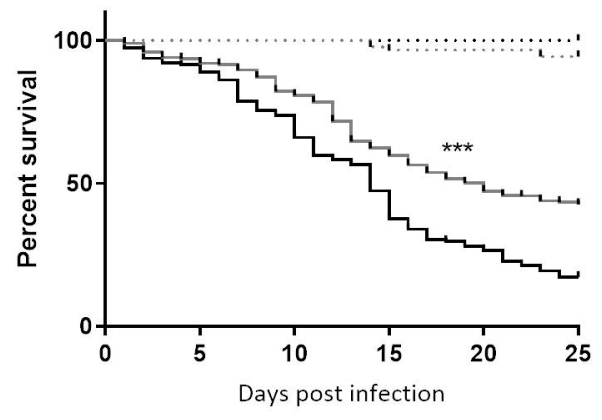

D

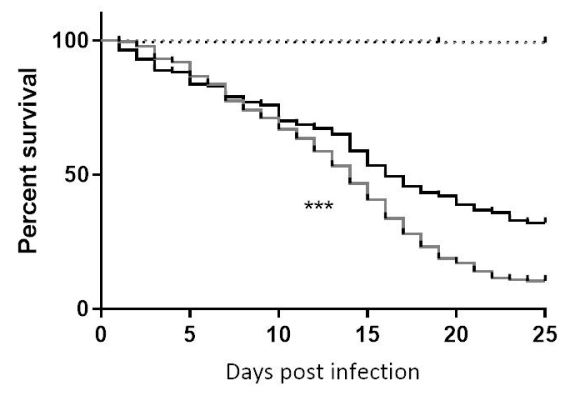

C ImpL2 knockdown - pathogen load of L. monocytogenes

24 hours post infection

$$
12 \text { days post infection }
$$
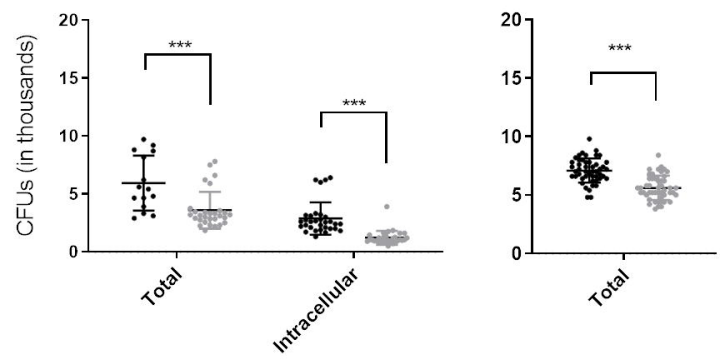

Hml>Gal4; TubGal80 ${ }^{\text {TS }} x$

- TRiP ${ }^{\text {control }}$

- $I m p L 2^{R N A i}$

E ImpL2 overexpression - pathogen load of L. monocytogenes 24 hours post infection 12 days post infection
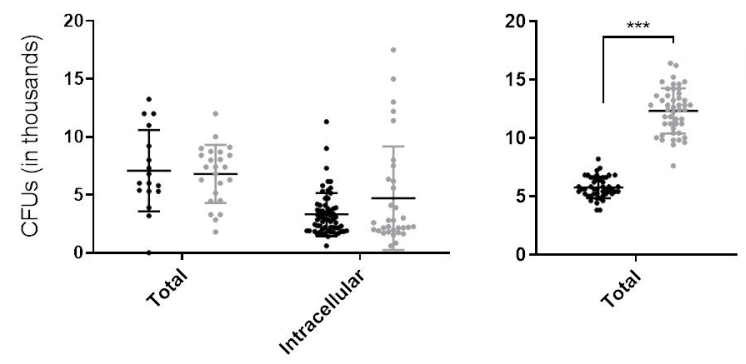

Hml>Gal4; TubGal80 ${ }^{\text {TS }} x$

- $w^{1118}$

ImpL2 ${ }^{\mathrm{CDS}}$
Hml>Gal4; TubGal80 $0^{\text {TS }} \mathrm{x}$

LL2 $/$ L.m.

- $W^{1118} /$ L.m. infected

a. $W^{1118} /$ Buff. injected
$\mathrm{Hml}>$ Gal4; TubGal80 ${ }^{\text {TS }} \mathbf{x}$

- ImpL2 ${ }^{\mathrm{RNAi}} /$ L.m. infected

- $\mathrm{TRiP}^{\mathrm{control}} /$ L.m. infected

. . TRiP $\mathrm{T}^{\text {control} / \text { Buff. injected }}$ 


\section{control}

THP-1 cells

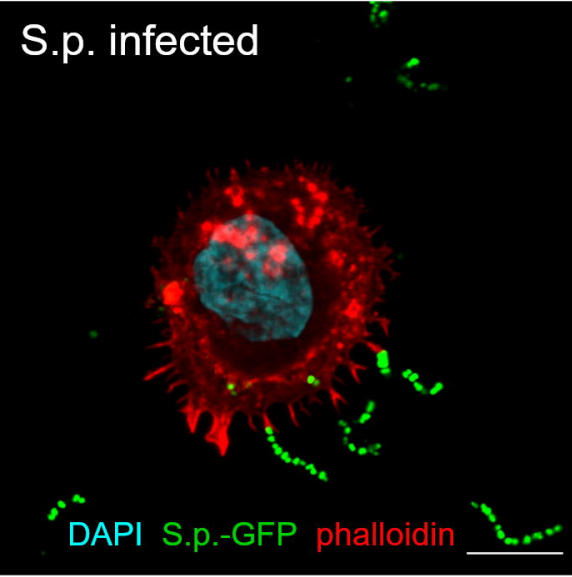

B

Expression of IGFBPs in THP-1 cells

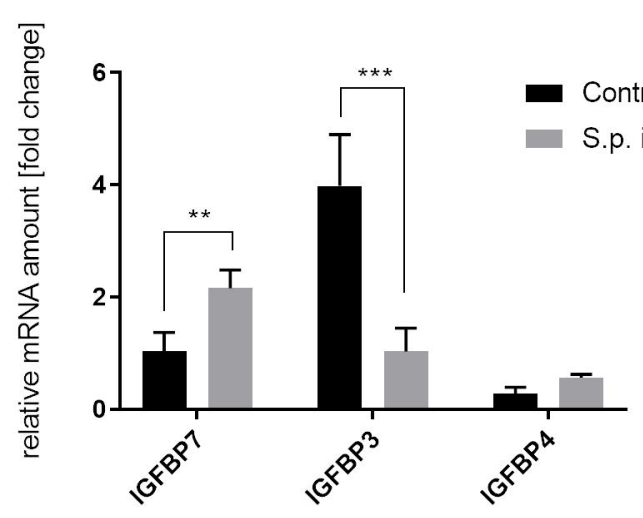




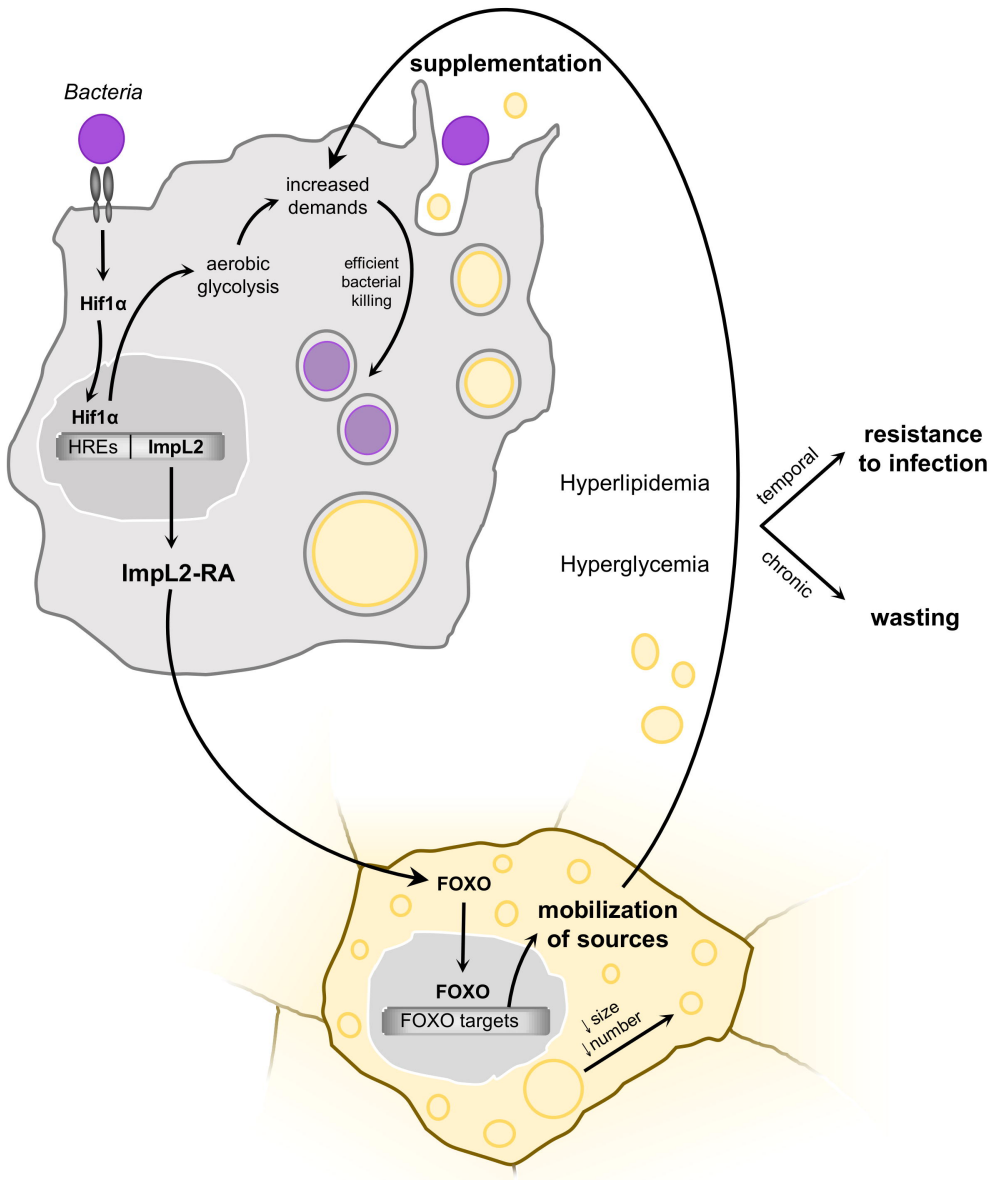

